\title{
Assembly of $\alpha$-Glucan by GlgE and GlgB in Mycobacteria and Streptomycetes
}

\author{
Abdul M. Rashid, ${ }^{\dagger}$ Sibyl F. D. Batey, ${ }^{\dagger}$ Karl Syson, ${ }^{\dagger}$ Hendrik Koliwer-Brandl, $^{\#}$ Farzana Miah, $^{\dagger}$ \\ J. Elaine Barclay, ${ }^{\ddagger}$ Kim C. Findlay, ${ }^{\ddagger}$ Karol P. Nartowski, ${ }^{\S}$ Yaroslav Z. Khimyak, ${ }^{\S}$ Rainer Kalscheuer, ${ }^{\#}$ \\ and Stephen Bornemann*, ${ }^{*}$ \\ ${ }^{\dagger}$ Biological Chemistry Department, John Innes Centre, Norwich Research Park, Norwich NR4 7UH, United Kingdom \\ ${ }^{\#}$ Institute for Medical Microbiology and Hospital Hygiene, and Institute for Pharmaceutical Biology and Biotechnology, \\ Heinrich-Heine-University Düsseldorf, Universitätsstrasse 1, 40225 Düsseldorf, Germany \\ ¥Cell and Developmental Biology Department, John Innes Centre, Norwich Research Park, Norwich NR4 7UH, United Kingdom \\ ${ }^{\S}$ School of Pharmacy, University of East Anglia, Norwich Research Park, Norwich NR4 7TJ, United Kingdom
}

\section{Supporting Information}

ABSTRACT: Actinomycetes, such as mycobacteria and streptomycetes, synthesize $\alpha$-glucan with $\alpha-1,4$ linkages and $\alpha-1,6$ branching to help evade immune responses and to store carbon. $\alpha$-Glucan is thought to resemble glycogen except for having shorter constituent linear chains. However, the fine structure of $\alpha$-glucan and how it can be defined by the maltosyl transferase GlgE and branching enzyme GlgB were not known. Using a combination of enzymolysis and mass spectrometry, we compared the properties of $\alpha$-glucan isolated from actinomycetes with polymer synthesized in vitro by GlgE and $\mathrm{GlgB}$. We now propose the following assembly mechanism. Polymer synthesis starts with GlgE and its donor substrate, $\alpha$-maltose 1-phosphate, yielding a linear oligomer with a degree of polymerization $(\sim 16)$ sufficient for GlgB to introduce a branch. Branching involves strictly intrachain transfer to generate a $\mathrm{C}$ chain (the only constituent chain to retain its reducing end), which now bears an A chain (a nonreducing end terminal branch that does not itself bear a branch). GlgE preferentially extends A chains allowing GlgB to act iteratively to generate new A chains emanating from B chains (nonterminal branches that themselves bear a branch). Although extension and branching occur primarily with A chains, the other chain types are sometimes extended and branched such that some B chains (and possibly C chains) bear more than one branch. This occurs less frequently in $\alpha$ glucans than in classical glycogens. The very similar properties of cytosolic and capsular $\alpha$-glucans from Mycobacterium tuberculosis imply GlgE and GlgB are sufficient to synthesize them both.
$\alpha$-Glucans are widespread among bacteria, yeasts, plants, insects, and mammals, and in most cases they are thought to act as a store of carbon and energy. Arguably the best documented $\alpha$-glucan in bacteria is glycogen, which typically accumulates under nitrogen-limiting growth conditions. ${ }^{1}$ Glycogen comprises linear $\alpha$-1,4-linked malto-oligosaccharides connected by $\alpha$-1,6-linked branch points giving rise to an arboreal (tree-like) dendrimer and particles tens of nanometers in diameter. In the classical bacterial glycogen pathway, the $\alpha$ 1,4-links are synthesized by glycogen synthase GlgA (E.C. 2.4.1.21), using ADP-glucose as the sugar donor (Figure 1), and the $\alpha-1,6$-linked branches are generated by GlgB branching enzyme (E.C. 2.4.1.18). About 32\% of sequenced bacterial genomes contain the genes coding for the enzymes of the classical pathway. ${ }^{2}$

The alternative GlgE $\alpha$-glucan pathway has been discovered recently, ${ }^{3,4}$ which is about half as widespread among bacteria as the classical pathway. ${ }^{2}$ This involves trehalose synthase and maltose kinase that convert trehalose into $\alpha$-maltose 1 - phosphate (Figure 1), which is the donor for the maltosyl transferase GlgE (E.C. 2.4.99.16) that creates $\alpha$-1,4-linkages. ${ }^{5}$ Bioinformatic analysis suggests that even when organisms possess both the classical and GlgE pathways, they possess only one GlgB enzyme, ${ }^{2}$ implying the branching enzyme is shared between both pathways. GlgB branching enzymes associated with these pathways are members of the glycoside hydrolase 13_9 CAZy family. ${ }^{6,7}$ Interestingly, the gene for GlgB is often clustered with the gene for GlgE rather than for GlgA in such cases. Some organisms possess only the GlgE pathway, and we have established using genetics that GlgE and $G \operatorname{lgB}$ are necessary and sufficient for the biosynthesis of $\alpha$-glucan in one such organism, the actinomycete Streptomyces venezuelae. ${ }^{8}$

The GlgE pathway is widespread among other actinomycetes such as mycobacteria. ${ }^{2}$ Indeed, both GlgE and GlgB have been

Received: March 7, 2016

Revised: $\quad$ May 23, 2016

Published: May 25, 2016 


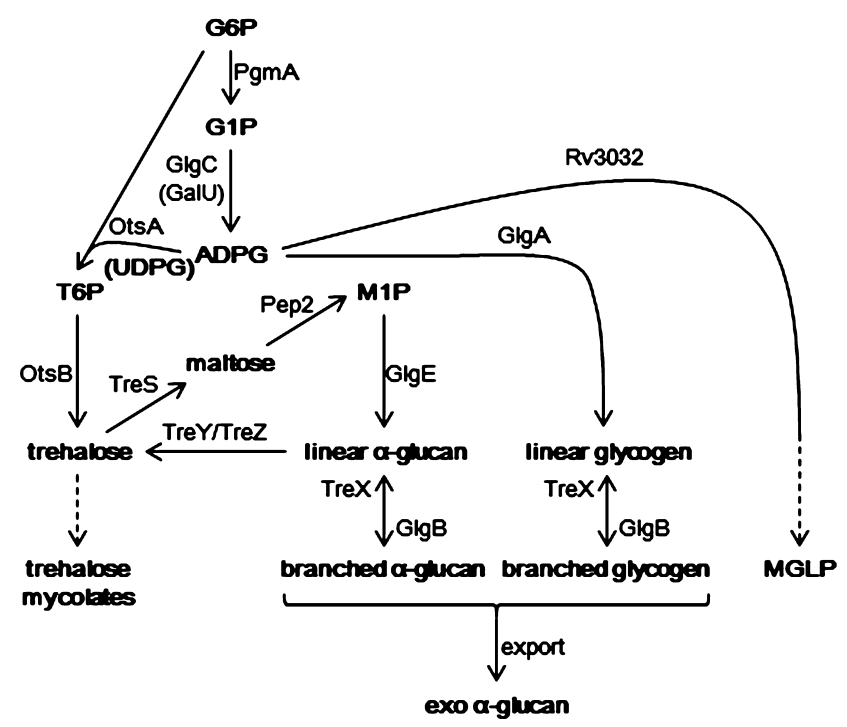

Figure 1. Metabolism of $\alpha$-glucans thought to exist in Mycobacterium tuberculosis. G6P, glucose 6-phosphate; G1P, glucose 1-phosphate; M1P, $\alpha$-maltose 1-phosphate; T6P, trehalose 6-phosphate; ADPG, ADP-glucose; UDPG, UDP-glucose; MGLP, methylglucose lipopolysaccharide; PgmA, phosphoglucomutase; GlgC, ADP-glucose pyrophosphorylase; GalU, UDP-glucose pyrophosphorylase; OtsA, trehalose 6-phosphate synthase; OtsB, trehalose 6-phosphate phosphatase; TreS, trehalose synthase; Pep2, maltose kinase; GlgE, maltosyl transferase; GlgB, branching enzyme; TreX, debranching enzyme; TreY, maltooligosyltrehalose synthase; TreZ, maltooligosyltrehalose trehalohydrolase; GlgA, glycogen synthase; Rv3032, putative glycosyltransferase.

genetically validated as potential drug targets for the treatment of tuberculosis. ${ }^{3}$ Some species, including Mycobacterium tuberculosis, possess not only the genes for the GlgE and classical pathways, but also genes for a methyl glucose lipopolysaccharide pathway (Figure 1). This pathway seems to involve $\mathrm{Rv} 3032$ in the formation of $\alpha-1,4$ linkages in the highly modified malto-oligosaccharide product of this pathway. ${ }^{9}$ This specialized $\alpha$-glucan is thought to chaperone fatty acid biosynthesis. Carbohydrate metabolism in mycobacteria is further complicated by the fact that the capsule coating the cells is mainly composed of a glycogen-like $\alpha$-glucan. ${ }^{10,11}$ This polymer might contribute to virulence of $M$. tuberculosis by binding to DC-SIGN to evade the host immune response. ${ }^{12-15}$ The capsular and cytosolic $\alpha$-glucan polymers appear to be very similar, with only subtle differences having been reported. ${ }^{15,16}$ Quite how each metabolic pathway contributes to each $\alpha$ glucan product is not yet clear.

Mycobacterial $\alpha$-glucans appear to resemble classical glycogen except for a higher degree of branching and correspondingly shorter linear chains, ${ }^{10,16,17}$ This implies that the branching enzyme in mycobacteria makes shorter branches than the corresponding enzymes in organisms that synthesize classical glycogen, such as in Escherichia coli. ${ }^{18}$ To address this issue, we first characterized GlgE-derived $\alpha$-glucan from $S$. venezuelae, and $\alpha$-glucans from mycobacteria. We then determined the chain length specificity of actinomycete GlgB branching enzymes and the chain specificity of $M$. tuberculosis GlgE. This enabled us to establish how the GlgE pathwayderived polymer is assembled, which has implications for how the cytosolic and capsular $\alpha$-glucans of $M$. tuberculosis are synthesized.

\section{MATERIALS AND METHODS}

Materials. Dextrans (5, 12, 25, 50, 100, 150, 270, 410, and $1400 \mathrm{kDa}$ ), amylose from potatoes (average mass $>150 \mathrm{kDa}$ ), amylopectin from potato, individual malto-oligosaccharides (degree of polymerization, DP2-7), rabbit liver glycogen and pullulanases were purchased from Sigma-Aldrich (Gillingham, United Kingdom). Malto-octaose (DP8) was purchased from Carbosynth (Compton, United Kingdom). Amylose 2800 (mean DP $12.6 \pm 5.3$, median and mode DP11, DP6-16 contributing at least $5 \%$ of total malto-oligosaccharides according to capillary electrophoresis) was purchased from TCI Europe N.V. (Oxford, United Kingdom). $\alpha$-Maltose 1phosphate was synthesized as previously described. ${ }^{19}\left[\mathrm{U}-{ }^{13} \mathrm{C}\right] \alpha$ maltose 1-phosphate was a gift from John (Jack) Thompson.

Isolation of $\boldsymbol{\alpha}$-Glucans. Streptomyces venezuelae. ATCC 10712 was grown on sterile cellophane discs on MYM Tap agar plates at $30{ }^{\circ} \mathrm{C}$ for $30 \mathrm{~h}$. The cells were removed from the cellophane, suspended in water $(15 \mathrm{~mL})$, and centrifuged at $4000 \mathrm{~g}$ for $30 \mathrm{~min}$. The cells were washed three more times, suspended in water $(10 \mathrm{~mL})$, and disrupted by sonication. The cell debris was pelleted by centrifugation at $30000 \mathrm{~g}$ for $15 \mathrm{~min}$, and the resultant supernatant was washed twice with a $1: 1(\mathrm{v} /$

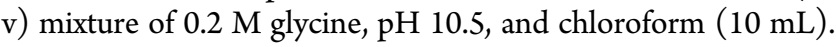
The aqueous layer was concentrated to $\sim 8 \mathrm{~mL}$ using a rotary evaporator and then centrifuged at $108000 \mathrm{~g}$ at $4{ }^{\circ} \mathrm{C}$ for $2 \mathrm{~h}$. The gelatinous pellet was collected and dissolved in water ( 2 $\mathrm{mL})$, and the $\alpha$-glucan was precipitated with ethanol (3 vol) at $4{ }^{\circ} \mathrm{C}$. The solid collected by centrifugation at $4000 \mathrm{~g}$ for $10 \mathrm{~min}$ was redissolved in water $(8 \mathrm{~mL})$ and centrifuged at $12000 \mathrm{~g}$ for $10 \mathrm{~min}$ to remove insoluble material. The ultracentrifugation and ethanol precipitation steps were repeated. Finally the pellet was dissolved in $2 \mathrm{~mL}$ of water and freeze-dried to yield the $\alpha$ glucan as an amorphous white powder.

Mycobacterium smegmatis. Cells were grown on sterile cellophane discs for $72 \mathrm{~h}$ at $37{ }^{\circ} \mathrm{C}$ on Difco Middlebrook $7 \mathrm{H} 10$ agar solid medium supplemented with $10 \% \operatorname{ADS}(5 \% \mathrm{w} / \mathrm{v}$ bovine serum albumin, $2 \%$ glucose, and $0.85 \% \mathrm{NaCl}$ ). Cells were resuspended in $10 \mathrm{~mL}$ of water and disrupted by sonication. $\alpha$-Glucan was extracted as described above.

Escherichia coli. BL21(DE3) cells were transformed with a pUC19 vector conferring resistance to carbenicillin. Cells were grown at $37{ }^{\circ} \mathrm{C}$ for $22 \mathrm{~h}$ in nitrogen-limited $\mathrm{M} 9$ medium $(1 \mathrm{~L})$ comprising $\mathrm{Na}_{2} \mathrm{HPO}_{4} \cdot 7 \mathrm{H}_{2} \mathrm{O}(6 \mathrm{~g}), \mathrm{KH}_{2} \mathrm{PO}_{4}(3 \mathrm{~g}), \mathrm{NaCl}(0.5$ g), $\mathrm{pH} 7.4$, glucose $(3 \mathrm{~g}),\left(\mathrm{NH}_{4}\right)_{2} \mathrm{SO}_{4}(0.22 \mathrm{~g}), \mathrm{MgSO}_{4} \cdot 7 \mathrm{H}_{2} \mathrm{O}$ $(0.25 \mathrm{~g}), \mathrm{CaCl}_{2} \cdot 2 \mathrm{H}_{2} \mathrm{O}(15 \mathrm{mg})$, and thiamine $(10 \mathrm{mg})$, supplemented with carbenicillin $(100 \mathrm{mg})$. Cell were harvested by centrifugation and resuspended in $10 \mathrm{~mL}$ of water. Glycogen was isolated as described for $\alpha$-glucan above.

M. tuberculosis. H37Rv cells were grown for 2 weeks in 100 $\mathrm{mL}$ cultures in a bovine serum albumin-free minimal medium containing $20 \mathrm{mM}$ glucose, $0.5 \%$ glycerol, and $0.05 \%$ tyloxapol. ${ }^{20}$ Because of the loose attachment of the $M$. tuberculosis capsule layer to the cell surface, treatment with detergent and physical agitation were sufficient to quantitatively strip off the capsule from cells and thus allow the satisfactory separation of capsular and cytosolic $\alpha$-glucan polymers. ${ }^{21}$ The cells were harvested by centrifugation. The supernatant was sterilized by passing twice through a $0.22 \mu \mathrm{m}$ sterile filter, heat inactivated at $65{ }^{\circ} \mathrm{C}$ for $12 \mathrm{~h}$, and concentrated to $1.5 \mathrm{~mL}$ by centrifugation $(4000 \mathrm{~g})$ through a $30 \mathrm{kDa}$ cutoff membrane filtration device. The retentate was diluted with water $(10 \mathrm{~mL})$ and concentrated to $1.5 \mathrm{~mL}$. The concentrate was treated with 
absolute ethanol ( $5 \mathrm{vol}$ ), and the resulting precipitate was collected by centrifugation. The solid was dissolved in water (1 $\mathrm{mL}$ ) and centrifuged, and the resulting supernatant was freezedried to yield capsular $\alpha$-glucan as an amorphous solid. The cell pellet was washed twice with $20 \mathrm{mM}$ sodium phosphate buffer, $\mathrm{pH} 7.4$, containing $20 \mathrm{mM} \mathrm{NaCl}$ to remove all remaining capsular material, resuspended in water, and heated for $4 \mathrm{~h}$ at $95^{\circ} \mathrm{C}$. The cell extract was centrifuged to remove debris, and the supernatant was centrifuged at $108000 \mathrm{~g}$ at $4{ }^{\circ} \mathrm{C}$ for $2 \mathrm{~h}$. The gelatinous pellet was dissolved in water $(0.5 \mathrm{~mL})$, and the $\alpha$ glucan was precipitated with ethanol (3 vol). The solid was collected by centrifugation, redissolved in water $(0.5 \mathrm{~mL})$, and freeze-dried to yield cytosolic $\alpha$-glucan as an amorphous power.

NMR Spectroscopy. Solution-state NMR spectra were recorded at $298 \mathrm{~K}$ on a Bruker Avance III $400 \mathrm{MHz}$ spectrometer and analyzed using Bruker TopSpin 2.1 (Rheinstetten, Germany). Chemical shifts in $\mathrm{D}_{2} \mathrm{O}$ are reported with reference to residual water at $\delta_{\mathrm{H}} 4.79$ or to the methyl resonance of internal acetone at $\delta_{\mathrm{C}} 29.84 \mathrm{ppm}$. Assignments were made with the aid of COSY and HSQC experiments. The assignment of the peaks was based on our previous studies. ${ }^{3,22}$

Pulse field gradient DOSY experiments ${ }^{23}$ were carried out with up to $5 \mathrm{mg}$ of polymer in $0.6 \mathrm{~mL}$ of $\mathrm{D}_{2} \mathrm{O}$ at $313 \mathrm{~K}$ using TopSpin 2.1. The gradient strength was incremented longitudinally in 16 steps from $2 \%$ up to $95 \%$ of the maximum gradient strength. Diffusion times and gradient pulse durations were optimized for each experiment in order to achieve a $95 \%$ decrease in the resonance intensity at the largest gradient amplitude. Typically, diffusion time was set at $200 \mu \mathrm{s}$ and the diffusion gradient length at $3.5 \mathrm{~ms}$. The $2 \mathrm{D}$ diffusion spectra were analyzed with $\mathrm{T} 1 / \mathrm{T} 2$ processing to derive the corresponding diffusion coefficient. The relationship between diffusion coefficient and molecular mass was determined using a series of dextrans (Figure S2).

Molecular radii were calculated from the Stokes-Einstein equation, which relates the diffusion coefficient $D$ to the macromolecular hydrodynamic radius $r$. The assumption that the macromolecules are spherical is reasonable given the morphology of particles according to electron microscopy (Figure 2).

$$
D=\frac{k_{\mathrm{B}} T}{6 \pi \eta r}
$$

where $k_{\mathrm{B}}=1.38 \times 10^{-23} \mathrm{~J} \mathrm{~K}^{-1}, T=313 \mathrm{~K}$, and dynamic viscosity of water $\eta=0.000653 \mathrm{~N} \mathrm{~s} \mathrm{~m}^{-2}$

(http://www.engineeringtoolbox.com/water-dynamickinematic-viscosity-d_596.html)

Solid-state NMR measurements were performed at $293 \mathrm{~K}$ using a Bruker $400 \mathrm{MHz}$ Avance III spectrometer equipped with a triple resonance probe at frequencies $400.23\left({ }^{1} \mathrm{H}\right)$ and $100.64 \mathrm{MHz}\left({ }^{13} \mathrm{C}\right)$. Amylose, amylopectin, and rabbit liver glycogen were each packed in $4 \mathrm{~mm}$ zirconia rotors, and $\alpha$ glucan samples were packed into $12 \mu \mathrm{L}$ CRAMPS rotors $4 \mathrm{~mm}$ in diameter. Samples were rotated at a magic angle spinning rate of $10 \mathrm{kHz} .{ }^{1} \mathrm{H}-{ }^{13} \mathrm{C}$ cross-polarization magic angle spinning (CP/MAS) spectra were acquired using the following parameters: ${ }^{1} \mathrm{H} \pi / 2$ pulse length $3.5 \mu \mathrm{s},{ }^{13} \mathrm{C} \pi / 2$ pulse length $3.5 \mu \mathrm{s}$, and ${ }^{1} \mathrm{H}-{ }^{13} \mathrm{C}$ CP contact time $2 \mathrm{~ms}$. SPINAL64 decoupling was used during signal acquisition with recycle delay of 20 s. The Hartmann-Hahn conditions for ${ }^{1} \mathrm{H}-{ }^{13} \mathrm{C}$ CP/MAS NMR experiment were set with hexamethylbenzene. Typically, 256 scans were acquired for amylose, amylopectin,

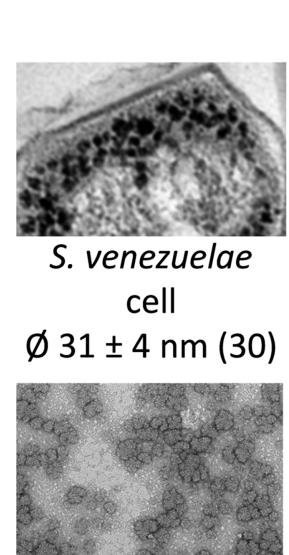

S. venezuelae $\alpha$-glucan

$\varnothing 42 \pm 3$ nm (10)

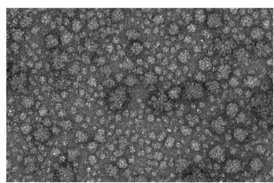

synthetic

$\alpha$-glucan

$\varnothing 32 \pm 2$ nm (48)

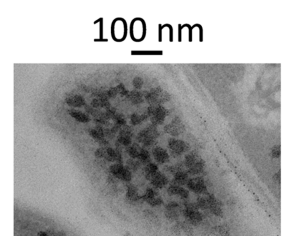

M. smegmatis cell
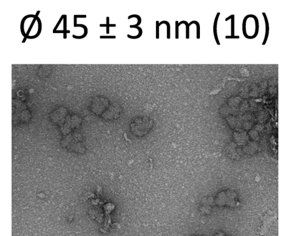

M. tuberculosis cytosolic $\alpha$-glucan $\varnothing 58 \pm 2 \mathrm{~nm}$ (10)

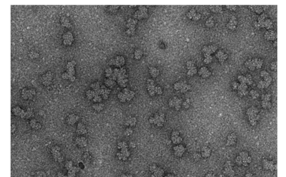

rabbit liver

glycogen

$\varnothing 38 \pm 2$ nm (10)

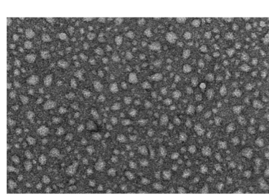

dextran 100

$\varnothing 23 \pm 3$ nm (10)

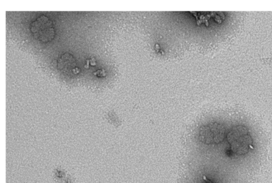

M. tuberculosis

capsular $\alpha$-glucan $\varnothing 63 \pm 3 \mathrm{~nm}$ (10)

Figure 2. Transmission electron microscopy of $\alpha$-glucans, glycogen, and dextran. Cells, isolated $\alpha$-glucans, and dextran 100 were stained using periodic acid-thiocarbohydrazide-silver proteinate (PATAg), uranyl acetate, and phosphotungstic acid, respectively. Representative images are shown. The scale bar represents $100 \mathrm{~nm}$. The diameters $(\varnothing)$ of the particles ( $\beta$ particles in the case of $\alpha$-glucans) are quoted as mean $\pm \operatorname{SE}(n)$. $\alpha$-Glucan isolated from $M$. smegmatis had a diameter of $35 \pm 3(21)$. Synthetic $\alpha$-glucan was produced by treating maltohexaose with $M$. tuberculosis GlgE and GlgB together with $\alpha$ maltose 1-phosphate. See main text and Table 1 for other measured diameters.

and rabbit liver glycogen and 2048 scans for $\alpha$-glucan. The ${ }^{13} \mathrm{C}$ chemical shifts were recorded with respect to trimethylsilane.

Transmission Electron Microscopy. Aqueous polymer samples (typically $0.1 \mathrm{mg} \mathrm{mL}^{-1}$ ) were allowed to settle briefly onto a 400 mesh copper grid with a carbon-coated pyroxylin support film before being negatively stained. Glycogen and $\alpha$ glucan was stained with $2 \%$ aqueous uranyl acetate, $\mathrm{pH} 4.5$, and dextran was stained with $1 \%$ phosphotungstic acid. The grids were viewed in a FEI Tecnai 20 transmission electron microscope (Eindhoven, Netherlands) at $200 \mathrm{kV}$, and digital TIFF images were taken using an AMT XR60B digital camera (Deben, Bury St Edmunds, UK).

S. venezuelae was grown for 5 days on MYM-TAP solid medium at $28{ }^{\circ} \mathrm{C},{ }^{24}$ and $M$. smegmatis was grown on Difco Middlebrook $7 \mathrm{H} 10$ agar solid medium supplemented with $10 \%$ ADS $(5 \% \mathrm{w} / \mathrm{v}$ bovine serum albumin, $2 \%$ glucose, and $0.85 \%$ $\mathrm{NaCl}$ ) for 3 days at $37^{\circ} \mathrm{C}$. Single colonies of $S$. venezuelae and M. smegmatis were positively stained for $\alpha$-glucan using periodic acid-thiocarbohydrazide-silver proteinate (PATAg) as previously described. ${ }^{8}$

Dynamic Light Scattering. Dynamic light scattering analysis was performed on a DynaPro Titan (Wyatt Technology Corporation, Santa Barbara CA, U.S.A.) equipped with DYNAMICS software (v6) using a $12 \mu \mathrm{L}$ sample cell at 
$293 \mathrm{~K}$. The sample concentration was typically $1 \mathrm{mg} \mathrm{mL} \mathrm{m}^{-1}$ in water.

lodine Complexes of $\boldsymbol{\alpha}$-Glucans. Lugol solution $(1 \mathrm{mg}$ $\mathrm{mL}^{-1} \mathrm{I}_{2}$ and $0.1 \mathrm{mg} \mathrm{mL}^{-1} \mathrm{KI}$; typically $0.5 \mathrm{vol}$ ) was added to samples of isolated polysaccharides, with further dilution in water as appropriate, and absorbance was measured either using a PerkinElmer Lambda 25 spectrophotometer (Coventry, United Kingdom) or a BMG Labtech CLARIOstar microtiter plate spectrophotometer (Ortenberg, Germany).

Reduction and Debranching of $\boldsymbol{\alpha}$-Glucans. Saccharides were reduced by adding aqueous $\mathrm{NaBH}_{4}$ (typically $1 \mathrm{mg}$ in 5 $\mu \mathrm{L}$ ) to aqueous samples (in $25 \mu \mathrm{L}$ ). The mixtures were incubated at $21{ }^{\circ} \mathrm{C}$ for $1 \mathrm{~h}$, acidified with dilute acetic acid, and treated with absolute ethanol $(5 \mathrm{vol})$. The resulting precipitated material was isolated by centrifugation and dried.

Polymer samples (typically in $25 \mu \mathrm{L}$ ) were debranched using Pseudomonas sp. isoamylase (with typically $2 \mu \mathrm{L}$ of a $1000 \mathrm{U}$ $\mathrm{mL}^{-1}$ stock solution) from Megazyme (Bray, Ireland) in 100 $\mathrm{mM}$ sodium acetate buffer, $\mathrm{pH} 4.0$, at $37{ }^{\circ} \mathrm{C}$ for $3 \mathrm{~h}$ (as illustrated in Figure 3A). NMR spectroscopy confirmed the loss of $\alpha-1,6$ linkages. Reactions were terminated by heating at 95 ${ }^{\circ} \mathrm{C}$ for $5 \mathrm{~min}$, and the products were analyzed either directly or after precipitation with ethanol (5 vol). Precipitated samples were dissolved in water $(50 \mu \mathrm{L})$ and freeze-dried to yield an amorphous powder.

Mass Spectrometry. Matrix-assisted laser desorption/ ionization mass spectra were acquired as described previously ${ }^{3}$ on Bruker Ultraflex or Autoflex Speed MALDI-TOF/TOF mass spectrometers (Coventry, United Kingdom), equipped with a pulsed nitrogen laser emitting at $337 \mathrm{~nm}$ or SmartbeamII $2 \mathrm{kHz}$ laser, respectively. Samples were typically mixed 1:2 v/ $\mathrm{v}$ with a $20 \mathrm{mg} \mathrm{mL}^{-1}$ solution of 2,5-dihydroxybenzoic acid in $50 \%$ aqueous acetonitrile and spotted on a target plate (Bruker MTP 384 Polished Steel TF Target). Data were processed using Bruker flexAnalysis software. The relative abundance of malto-oligosaccharides of DP $\geq 4$ was determined at least in duplicate by measuring the area under the peaks of all isotopes associated with each species of a given degree of polymerization using the Sophisticated Numerical Annotation Procedure with sugar-sodium adduct average composition. When experiments involved quantifying both reduced and nonreduced maltooligosaccharides in a sample, the intensity of the main isotopologue was used to quantify each species, taking into account the contribution of any nonreduced isotopologues with +2 Da when quantifying reduced species of the same mass. The percentage contribution of such nonreduced isotopologues for each DP of malto-oligosaccharide was determined from experimental spectra of authentic compounds. Any difference between the ionization efficiencies of reduced and nonreduced malto-octoase was $<15 \%$ in control spectra (data not shown), showing that quantitative comparison between such species is possible. Ion mobility mass spectrometry was carried out as described previously. ${ }^{25}$ Samples were analyzed using a Synapt G2 HDMS mass spectrometer (Waters Corp., Manchester, UK) using electrospray ionization in traveling-wave mobility mode with nitrogen and helium gas flows in negative mode.

Capillary Electrophoresis. Reducing saccharides were labeled with 8-aminopyrene-1,3,6-trisulfonic acid to facilitate their separation on the basis of charge and their detection by fluorescence spectrophotometry. Samples (typically $5 \mu \mathrm{L}$ ) were treated with 8-aminopyrene-1,3,6-trisulfonic acid $(1 \mu \mathrm{L}$ of 50 $\mathrm{mM}$ in $15 \%$ aqueous glacial acetic acid) and freshly prepared $\mathrm{NaCNBH}_{3}$ in tetrahydrofuran $(1 \mu \mathrm{L}$ of $1 \mathrm{M})$. Reaction

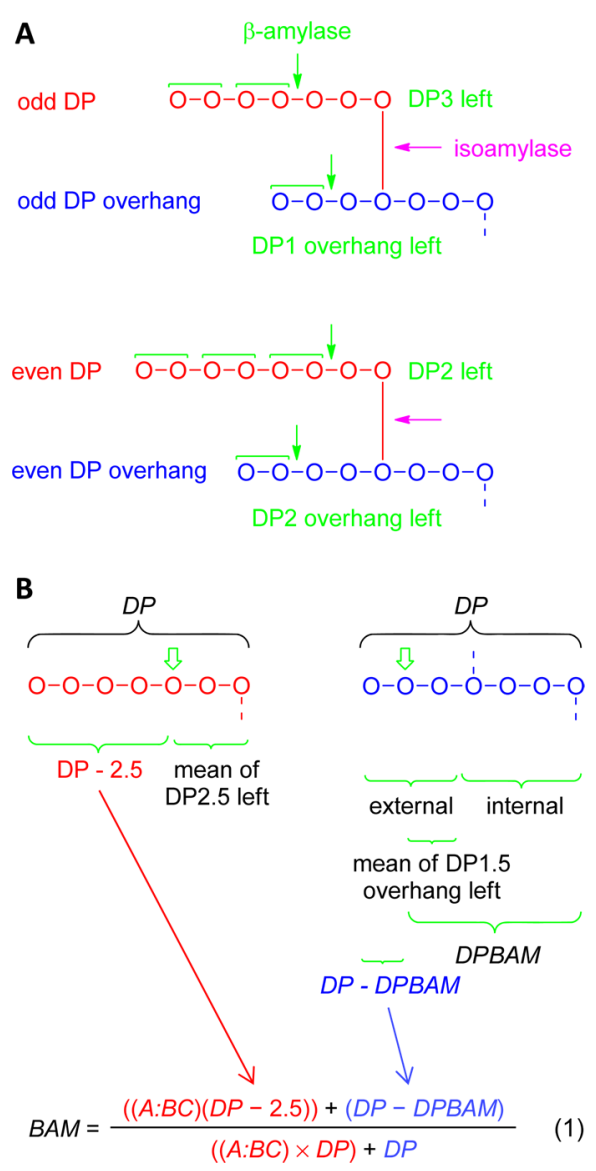

Figure 3. Cartoon showing the degradation of $\alpha$-glucan by $\beta$-amylase and isoamylase. (A) Isoamylase hydrolyzes the $\alpha$-1,6-linked branch points (indicated in purple). $\beta$-Amylase hydrolyses maltose units iteratively from the nonreducing ends of linear chains (indicated in green). A chains (shown in red) are left with a DP of either 3 or 2, depending on whether the original DP was odd or even, respectively. B chains (shown in blue) are left with an overhang of either DP 1 or 2 depending on whether the original overhang was odd or even, respectively. $\mathrm{C}$ chains are degraded in the same way as for $\mathrm{B}$ chains (not shown). (B) A chains are therefore extensively hydrolyzed by $\beta$ amylase leaving a mean of 2.5 glucosyl residues (i.e., DP2.5). B and C chains are hydrolyzed leaving a mean overhang of 1.5 glucosyl residues (indicated by the broad green arrows). The contribution of the hydrolysis of $\mathrm{A}$ and $\mathrm{B} / \mathrm{C}$ chains to the extent to which $\beta$-amylase degrades $\alpha$-glucan (BAM) is indicated in the context of eq 1 , which can be rearranged to give eq 2 that defines A:BC. Thus, the right-hand side of eq 1 , in both the numerator and the denominator, relates to the extent of hydrolysis of $B$ plus $C$ chains. The left-hand side relates to the extent of hydrolysis of the $\mathrm{A}$ chains, where $\mathrm{A}: \mathrm{BC}$ is the ratio of the numbers of $\mathrm{A}$ to $\mathrm{B}$ plus $\mathrm{C}$ chains normalized to the number of $\mathrm{B}$ plus C chains.

mixtures were incubated at $37^{\circ} \mathrm{C}$ for $4 \mathrm{~h}$, diluted 10 -fold with water. Samples were subjected to capillary electrophoresis using a Beckman Coulter PA800 ProteomeLab Protein Characterization System (High Wycombe, United Kingdom) using laserinduced fluorescence detection with an excitation wavelength of $488 \mathrm{~nm}$ and an emission band-pass filter of $520 \mathrm{~nm} \pm 10 \mathrm{~nm}$. An SCIEX N-CHO capillary (50 $\mu \mathrm{m}$ internal diameter $\times 50.2$ $\mathrm{cm} ; 40 \mathrm{~cm}$ effective length to detector) was used with a constant voltage of $-30 \mathrm{kV}$, the anode at the detector, and a temperature of $25{ }^{\circ} \mathrm{C}$. The capillary was rinsed with SCIEX N$\mathrm{CHO}$ buffer, and the samples were introduced at a pressure of 0.5 psi for $5 \mathrm{~s}$. 
Determination of the A:BC Chain Ratio. $\alpha$-Glucan and glycogen were each exhaustively treated for at least $1 \mathrm{~h}$ at $37^{\circ} \mathrm{C}$ with $\beta$-amylase from barley Megazyme (Bray, Ireland) in 10 $\mathrm{mM}$ MES $\mathrm{pH}$ 6.0. The only malto-oligosaccharide produced was maltose according to mass spectrometry as expected. The amount of maltose liberated by $\beta$-amylase was determined using the 3,5-dinitrosalicylic acid method. A solution of 3,5dinitrosalicylic acid (43.8 mM 3,5-dinitrosalicylic acid containing $1.06 \mathrm{M}$ potassium sodium $\mathrm{L}-(+)$-tartrate and $0.4 \mathrm{M} \mathrm{NaOH})$ was added to a sample $(1: 1 \mathrm{v} / \mathrm{v})$ with water and incubated at 95 ${ }^{\circ} \mathrm{C}$ for $5 \mathrm{~min}$. The mixtures were cooled on ice for $10 \mathrm{~min}$, diluted 2-fold, and the absorbance of the mixtures was measured at $540 \mathrm{~nm}$ and compared with a glucose calibration curve. The amount of glucose residues liberated would have been 2 times the amount of maltose detected. In separate experiments, all glucosyl residues within a polymer were liberated with Bacillus licheniformis $\alpha$-amylase (EC 3.2.1.1 in 50 $\mathrm{mM}$ MOPS buffer, $\mathrm{pH} 7.0$, at $99{ }^{\circ} \mathrm{C}$ for $6 \mathrm{~min}$ to generate shorter dextrins) followed by Aspergillus niger amyloglucosidase (EC 3.2.1.3 in $200 \mathrm{mM}$ sodium acetate buffer, $\mathrm{pH} 4.5$, at $50{ }^{\circ} \mathrm{C}$ for $30 \mathrm{~min}$ to hydrolyze all remaining $\alpha-1,4$ and $\alpha-1,6$ linkages) from the Megazyme Total Starch Assay kit K-TSTA 05/2008 (Bray, Ireland), ${ }^{26}$ allowing the total glucose content to be determined using 3,5-dinitrosalicylic acid. All measurements were carried out at least in duplicate. For example, three independent replicate experiments were carried out with $S$. venezuelae $\alpha$-glucan giving $21.0 \pm 4.7$ (SD of replicate means).

The proportion of glucose residues liberated as maltose from $\mathrm{A}$ and $\mathrm{B} / \mathrm{C}$ chains by $\beta$-amylase (BAM) is defined by eq 1 as illustrated in Figure 3.

$$
\mathrm{BAM}=\frac{((\mathrm{A}: \mathrm{BC})(\mathrm{DP}-2.5))+(\mathrm{DP}-\mathrm{DPBAM})}{((\mathrm{A}: \mathrm{BC}) \times \mathrm{DP})+\mathrm{DP}}
$$

where $\mathrm{A}: \mathrm{BC}$ is the ratio of the numbers of $\mathrm{A}$ to $\mathrm{B}$ plus $\mathrm{C}$ chains normalized to the number of $\mathrm{B}$ plus $\mathrm{C}$ chains (where, in this context, the sole $\mathrm{C}$ chain per molecule is equivalent to a $\mathrm{B}$ chain because it bears at least one branch also).

DP is the mean degree of polymerization of all chains (it is assumed that all types of chain have a similar mean DP; nevertheless, the lower the $\mathrm{A}: \mathrm{BC}$ chain ratio, the less sensitive eq 2 below becomes to differences in the mean DP of $A$ chains); e.g., five independent replicate experiments were carried out with $S$. venezuelae $\alpha$-glucan giving $7.3 \pm 0.4$ (SD of replicate means).

DPBAM is the mean degree of polymerization of linear B chains after digestion with $\beta$-amylase determined by mass spectrometry (where maltose and malto-triose were ignored because they were derived from A chains); e.g., four independent replicate experiments were carried out with $S$. venezuelae $\alpha$-glucan giving $6.5 \pm 0.2$ (SD of replicate means).

Equation 1 can be rearranged to define the $\mathrm{A}: \mathrm{BC}$ ratio to give eq 2

$$
\mathrm{A}: \mathrm{BC}=\frac{\mathrm{DP}-\mathrm{DPBAM}-(\mathrm{BAM} \times \mathrm{DP})}{(\mathrm{BAM} \times \mathrm{DP})-\mathrm{DP}+2.5}
$$

Production of Recombinant Enzymes. The DNA sequences of the $\operatorname{glg} B$ genes from $M$. tuberculosis $H 37 \mathrm{Rv}$, Mycobacterium smegmatis $\mathrm{mc}^{2} 155$, Streptomyces coelicolor A3(2) isoform 1, and Streptomyces venezuelae ATCC 10712 were synthesized with optimized codon usage for expression in Escherichia coli (Genscript Corporation, Piscataway, NJ, U.S.A.), allowing the production of each enzyme with an $\mathrm{N}$ - terminal $\mathrm{His}_{6}$ tag and TEV cleavage site. Each gene was flanked at the $5^{\prime}$ end by an NdeI restriction site and a sequence encoding a $\mathrm{His}_{6}$ tag and a TEV protease cleavage site, and at the $3^{\prime}$ end by a BamHI restriction site. Each gene, with exceptions described below, was subcloned in to a pET-21a(+) (Novagen, Watford, United Kingdom) vector using NdeI and $B a m H I$ restriction sites and heterologously expressed in E. coli BL21(DE3). The M. tuberculosis $\mathrm{H} 37 \mathrm{Rv} \operatorname{glgB}$ gene sequence was mutated into that of M. tuberculosis CCDC5180 by changing the codon for Ser470 into one for Pro using QuikChange (Agilent Technologies, Santa Clara, CA, U.S.A.). Cells containing the resulting expression plasmid were cultured in Lysogeny Broth containing carbenicillin $\left(100 \mu \mathrm{g} \mathrm{mL}^{-1}\right)$ at 37 ${ }^{\circ} \mathrm{C}$. When the cells reached an OD600 nm of 0.6 , expression was induced by the addition of $0.5 \mathrm{mM}$ isopropyl $\beta$-Dthiogalactopyranoside. After incubation at $30{ }^{\circ} \mathrm{C}$ for $2 \mathrm{~h}$ and then $16^{\circ} \mathrm{C}$ for $17 \mathrm{~h}$, the cells were harvested by centrifugation and resuspended in $50 \mathrm{mM}$ Tris- $\mathrm{HCl}, \mathrm{pH} 8.0$, containing 15 $\mathrm{mM}$ imidazole, $300 \mathrm{mM} \mathrm{NaCl}$, DNase 1, and a Complete protease inhibitor cocktail tablet (Roche, Burgess Hill, United Kingdom). A similar strategy was used for the production of the other recombinant proteins. The cells were disrupted with a TS Series Benchtop 1.1 kW cell disruptor (Constant Systems Ltd.) at $25 \mathrm{kPSI}$. The resulting cell lysate was separated from the cell debris by centrifugation at $20000 \mathrm{~g}$ for $15 \mathrm{~min}$ at $4{ }^{\circ} \mathrm{C}$. Enzyme were purified from each cell lysate by application onto a $1 \mathrm{~mL}$ FF HisTrap column (GE Healthcare, Amersham, United Kingdom). Protein was eluted with 15-300 mM imidazole gradient. Enzymes were further purified to remove any traces of E. coli amylase activities using a HiLoad 26/60 Superdex 200 gel filtration column (GE healthcare) equilibrated with $20 \mathrm{mM}$ sodium phosphate buffer, $\mathrm{pH} 7.4$, containing $20 \mathrm{mM} \mathrm{NaCl}$. The purified proteins were homogeneous according to SDS-PAGE with Coomassie staining.

The production of $S$. venezuelae GlgB was not successful with $\mathrm{pET} 21 \mathrm{a}$ vector. The $\lg \mathrm{lg}$ gene was ligated into a lower copynumber pBAD43 vector ${ }^{27}$ using $\mathrm{NcoI}$ and HindIII restriction sites, placing the gene under the more stringent control of an arabinose-inducible promoter. GlgB was produced in E. coli TOP10 cells (Novogen) grown at $37{ }^{\circ} \mathrm{C}$ to an OD at $600 \mathrm{~nm}$ of 0.6 in Lysogeny Broth before induction with $0.02 \% \mathrm{w} / \mathrm{v} \mathrm{L-}$ arabinose followed by a further $5 \mathrm{~h}$ of incubation. The enzyme was purified using both $\mathrm{Ni}$-affinity and size exclusion chromatographies as described above.

Enzyme-Catalyzed Reactions. To generate synthetic $\alpha$ glucan and to determine the branch length specificity of GlgB enzymes, $1 \mathrm{mM}$ malto-hexaose and $10.1 \mathrm{mM} \alpha$-maltose-1phosphate were incubated with $7 \mu \mathrm{g}$ each of recombinant GlgE and $\mathrm{GlgB}$ (giving $<2 \mu \mathrm{M}$ final concentrations) in $100 \mathrm{mM}$ bisTris propane, $\mathrm{pH} 7.0$, containing $50 \mathrm{mM}$ sodium chloride in a total volume of $50 \mu \mathrm{L}$. After $18 \mathrm{~h}$ at $22^{\circ} \mathrm{C}$, a second aliquot of $\alpha$-maltose-1-phosphate was added. After a further $90 \mathrm{~h}$, the sample was heated at $95{ }^{\circ} \mathrm{C}$ for $5 \mathrm{~min}$, cooled, and washed 5 times using a $10000 \mathrm{Da}$ cutoff membrane with $100 \mathrm{mM}$ sodium acetate buffer, $\mathrm{pH} 4.0$, to remove small maltooligosaccharides. The sample was analyzed by electron microscopy, mass spectrometry, or capillary electrophoresis with or without debranching as appropriate.

In other experiments, malto-oligosaccharides (typically $5 \mathrm{mg}$ ) were extended with $\operatorname{GlgE}(14 \mu \mathrm{g})$ and $6.6 \mathrm{mM} \alpha$-maltose 1 phosphate in a total volume of $122 \mu \mathrm{L}$ at $37{ }^{\circ} \mathrm{C}$ for $3 \mathrm{~h}$. The enzyme was denatured by heating to $96{ }^{\circ} \mathrm{C}$ for $5 \mathrm{~min}$. As appropriate, $\mathrm{GlgB}(70 \mu \mathrm{g})$ was then added, for example, and the 
Table 1. Properties of $\alpha$-Glucans and Glycogen

\begin{tabular}{|c|c|c|c|c|c|}
\hline & \multicolumn{2}{|c|}{ M. tuberculosis } & \multirow{2}{*}{$\frac{\text { S. venezuelae }}{\text { cytosolic }}$} & \multirow{2}{*}{$\frac{\text { rabbit liver }}{\text { glycogen }}$} & \multirow{2}{*}{$\frac{\text { E. coli }}{\text { glycogen }}$} \\
\hline & capsular & cytosolic & & & \\
\hline \multicolumn{6}{|c|}{ Size } \\
\hline TEM $\beta$ particle $(\mathrm{nm})^{a}$ & $63 \pm 7$ & $58 \pm 2$ & $42 \pm 3$ & $38 \pm 2$ & $34 \pm 5$ \\
\hline after $\beta$-amylase $\mathrm{e}^{a, b}$ & $\mathrm{nd}^{c}$ & $\mathrm{nd}^{c}$ & $42 \pm 3$ & $29 \pm 2$ & $\mathrm{nd}^{c}$ \\
\hline DLS $\beta$ particle $(\mathrm{nm})^{d}$ & $64 \pm 8$ & $64 \pm 5$ & $51 \pm 11$ & $59 \pm 10$ & $\mathrm{nd}^{c}$ \\
\hline after $\beta$-amylase ${ }^{b, d}$ & $\mathrm{nd}^{c}$ & $\mathrm{nd}^{c}$ & $54 \pm 1$ & $40 \pm 1$ & $\mathrm{nd}^{c}$ \\
\hline NMR diffusion coefficient $\left(\mathrm{m}^{2} \mathrm{~s}^{-1}\right)^{e}$ & $\mathrm{nd}^{c}$ & $\mathrm{nd}^{c}$ & $2.5 \times 10^{-12}$ & $2.9 \times 10^{-12}$ & $\mathrm{nd}^{c}$ \\
\hline $\operatorname{NMR} \alpha$ particle $(\mathrm{nm})^{e}$ & $\mathrm{nd}^{c}$ & $\mathrm{nd}^{c}$ & $\sim 280$ & $\sim 240$ & $\mathrm{nd}^{c}$ \\
\hline NMR $\alpha$ particle $(\mathrm{Da})^{e}$ & $\mathrm{nd}^{c}$ & $\mathrm{nd}^{c}$ & $\sim 5 \times 10^{6}$ & $\sim 4 \times 10^{6}$ & $\mathrm{nd}^{c}$ \\
\hline \multicolumn{6}{|c|}{ Linear Chain $\mathrm{DP}^{f}$} \\
\hline overall mean $(\mathrm{DP})$ & $7.0 \pm 2.0$ & $7.2 \pm 1.9$ & $7.3 \pm 1.8$ & $10.8 \pm 4.6$ & $11.0 \pm 2.9$ \\
\hline C mean ${ }^{g}$ & $\mathrm{nd}^{c}$ & $\mathrm{nd}^{c}$ & $9.1 \pm 2.5$ & $\mathrm{nd}^{c}$ & $\mathrm{nd}^{c}$ \\
\hline \multicolumn{6}{|c|}{ Extent of Branching from } \\
\hline overall mean $\mathrm{DP}^{h}$ & 0.14 & 0.14 & 0.14 & 0.09 & 0.09 \\
\hline NMR spectroscopy ${ }^{i}$ & $\mathrm{nd}^{c}$ & $\mathrm{nd}^{c}$ & 0.14 & 0.09 & $\mathrm{nd}^{c}$ \\
\hline \multicolumn{6}{|c|}{$\beta$-Amylase Treatment } \\
\hline mean DP $(\text { DPBAM })^{f}$ & $6.1 \pm 1.4$ & $6.2 \pm 1.8$ & $6.5 \pm 1.6$ & $8.6 \pm 3.5$ & $9.8 \pm 3.3$ \\
\hline residues liberated \% (BAM) & 31.1 & 33.0 & $21.0 \pm 4.7$ & 47.5 & 40.5 \\
\hline internal chain length ${ }^{j}$ & $4.6 \pm 1.4$ & $4.7 \pm 1.8$ & $5.0 \pm 1.6$ & $7.1 \pm 3.5$ & $8.3 \pm 3.3$ \\
\hline $\mathrm{A}: \mathrm{BC}$ chain ratio $^{k}$ & 0.6 & 0.6 & $0.2 \pm 0.2$ & 0.9 & 0.8 \\
\hline mean branches per $\mathrm{B} / \mathrm{C}$ chain ${ }^{l}$ & $\sim 1.6$ & $\sim 1.6$ & $\sim 1.2 \pm 0.2$ & $\sim 1.9$ & $\sim 1.8$ \\
\hline
\end{tabular}

${ }^{a_{T}}$ The diameters of individual particles were determined by transmission electron microscopy (TEM) from at least 10 measurements (see Figure 2).

${ }^{b}$ The size of glycogen particles after treatment with $\beta$-amylase. ${ }^{c}$ Not determined. ${ }^{d}$ The hydrodynamic diameter of the dominant species of $<100 \mathrm{~nm}$ in each sample was determined using dynamic light scattering where at least five measurements were made. ${ }^{e}$ The diffusion coefficient, hydrodynamic diameter, and molecular mass of the dominant large species assigned as $\alpha$ particles were determined using pulse field gradient NMR spectroscopy (see Figure S2 for the calibration curve). ${ }^{f}$ The DP mean (mass average) was determined for products with a DP $\geq 4$ using mass spectrometry (see Figure 4 for representative spectra). Chain length distributions are expressed as SD. ${ }^{g}$ The DP of $\mathrm{C}$ chains was determined as described in Figure 5. ${ }^{h}$ Extent of branching ( $\alpha-1,6$ linkages divided by the sum of $\alpha-1,6$ and $\alpha-1,4$ linkages) equates to the reciprocal mean branch length. ${ }^{i}$ Extent of branching was also determined by integrating the appropriate anomeric resonances in ${ }^{1} \mathrm{H}$ NMR spectra without solvent suppression of samples subjected to repeated freeze-drying and resolubilization in $\mathrm{D}_{2} \mathrm{O} .{ }^{j}$ Calculated from DPBAM minus 1.5 . ${ }^{k}$ The ratio of the numbers of A to B plus C chains normalized to the number of B plus C chains, from eq $2 .{ }^{l}$ Essentially A:BC plus one.

mixture was incubated at $30{ }^{\circ} \mathrm{C}$ for $2 \mathrm{~h}$. The enzyme was then denatured by heating and analyzed as described above.

The GlgE-dependent extension of acceptors with $\alpha$-maltose 1-phosphate was monitored using malachite green to detect the production of inorganic phosphate as described previously. ${ }^{3,19,28}$

\section{RESULTS}

Isolation of $\alpha$-Glucan Particles from Actinomycetes and Classical Glycogen Particles from E. coli. Before characterizing GlgB branching enzymes from actinomycetes, we characterized the $\alpha$-glucans produced by these bacteria together with classical glycogens to allow direct comparisons to be made. To this end, $\alpha$-glucans/glycogen were first isolated from the cytosols of $S$. venezuelae, $M$. tuberculosis (the default strain in this study is $\mathrm{H} 37 \mathrm{Rv}$ ), and E. coli, from the capsule of $M$. tuberculosis, and from whole cells of M. smegmatis. We included $S$. venezuelae because we have previously shown that this organism generates $\alpha$-glucan solely by the GlgE pathway. ${ }^{8}$ This contrasts with E. coli, which generates its glycogen only via the classical GlgA glycogen pathway. ${ }^{2,29}$ NMR spectra of the isolated materials gave characteristic resonances of $\alpha-1,4-$ and $\alpha$-1,6-linked glucose polymers (examples of solution- and solidstate NMR spectra of $S$. venezuelae $\alpha$-glucan are shown in Figure S1). ${ }^{17,30-32}$ Transmission electron microscopy showed that each of the isolated polymers comprised $\beta$ particles that sometimes aggregated into larger $\alpha$ particles (Figure 2 and Table 1). ${ }^{33}$ According to both transmission electron micros- copy and dynamic light scattering, the $\beta$ particles had diameters ranging from $\sim 30$ to $\sim 60 \mathrm{~nm}$, consistent with previous reports. ${ }^{15-17,30,34}$ Sectioned cells of $S$. venezuelae and $M$. smegmatis that were stained for $\alpha$-glucan showed the presence of cytosolic $\beta$ particles of a similar diameter $(\sim 30-45 \mathrm{~nm})$ to that of isolated material (Figure 2). Only faint speckled staining was observed on the exterior of $M$. smegmatis cells, suggesting the presence of either little or diffuse capsular $\alpha$-glucan in these growth conditions. Pulse field gradient NMR spectroscopy showed that isolated $S$. venezuelae $\alpha$-glucan and rabbit liver glycogen contained slowly diffusing $\alpha$ particles with diameters of $\sim 240-280 \mathrm{~nm}$, equating to $\sim 5 \times 10^{6} \mathrm{Da}$ assemblies comprising $\sim 30000$ glucosyl residues (Table 1 and Figure S2). Similar values have been reported for material isolated from $M$. smegmatis. $^{35}$

Actinomycete $\alpha$-Glucans Are Composed of Short Linear Chains of Length $\sim 7-8$. It has previously been reported that the $\alpha$-glucans of mycobacteria contain shorter linear chains ${ }^{10,15-17,30,34,36}$ than those of classical glycogen. ${ }^{29}$ In order to establish whether this was the case with $S$. venezuelae $\alpha$-glucan, the isolated material was treated with isoamylase to hydrolyze the $\alpha-1,6$ linkages (Figure 3A). Such a sample remained in solution and did not give strongly colored complexes with iodine according to absorbance spectroscopy (Figure S3), as would be expected with liberated linear chains having a degree of polymerization (DP) of $<15 .{ }^{37}$ Indeed the absorbance of the iodine complexes was only slightly greater than with malto-heptaose. By contrast, debranched rabbit liver glycogen yielded a little precipitate in the absence of iodine, 

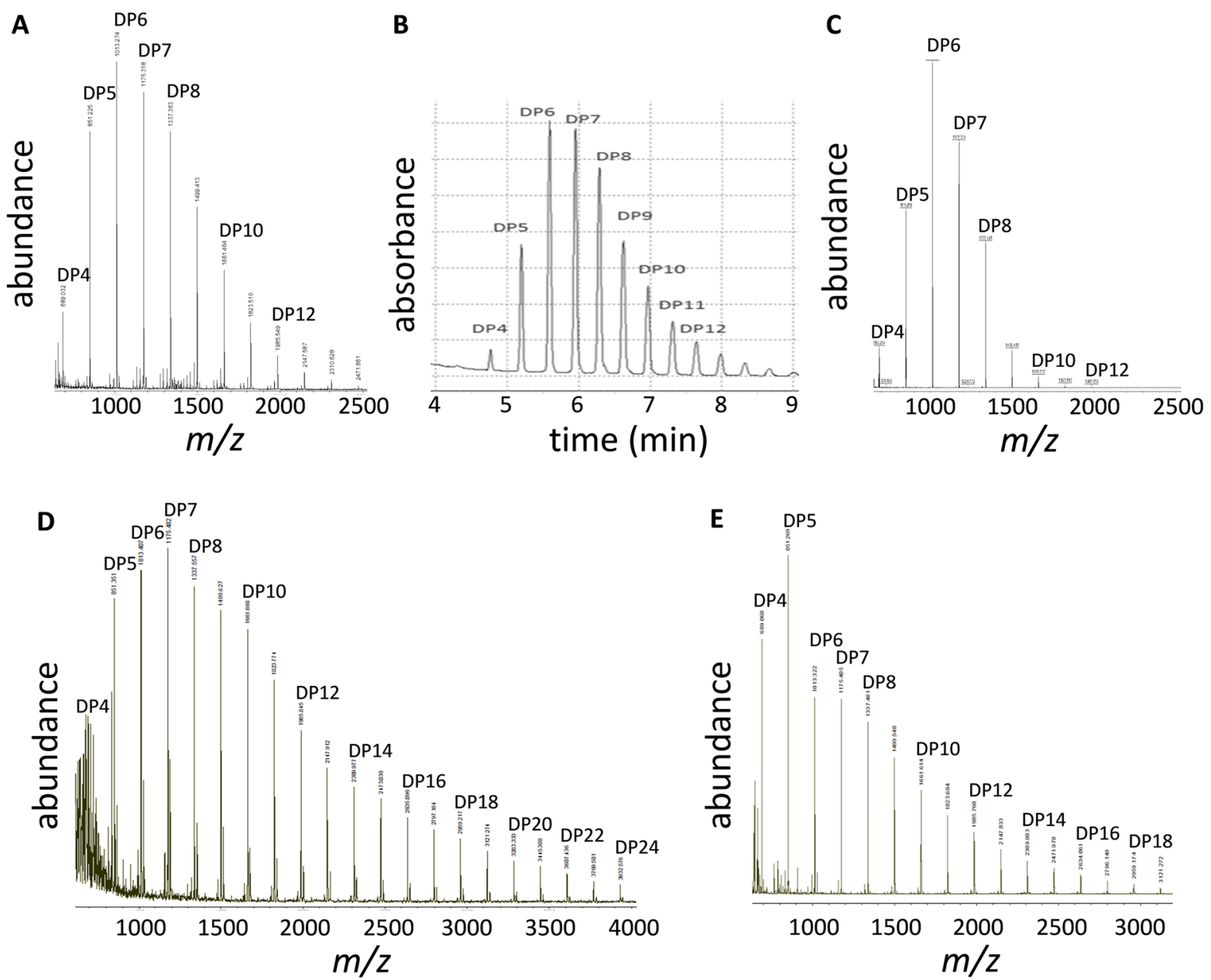

Figure 4. Branch length distribution of S. venezuelae $\alpha$-glucan and rabbit liver glycogen. The $\alpha-1,6$ linkages in the $\alpha$-glucan were hydrolyzed using isoamylase to $<1 \%$ according to ${ }^{1} \mathrm{H}$ NMR spectroscopy and subjected to either (A) MALDI MS or (B) capillary electrophoresis after treatment with 8-aminopyrene-1,3,6-trisulfonic acid and $\mathrm{NaCNBH}_{3}$, showing similar chain length distributions. Only after treatment with isoamylase were maltooligosaccharides observed (data not shown). (C) A sample treated with $\beta$-amylase before debranching was analyzed by MALDI-MS and showed a loss of longer DPs and a relative gain of medium DPs of $\sim 6$. Mass spectrometry of a sample that was treated solely with $\beta$-amylase showed the presence of only maltose as expected (data not shown). Debranched rabbit liver glycogen was subjected to MALDI MS either (D) without or (E) with pretreatment with $\beta$-amylase, leading to the substantial net reduction in DP. Only after treatment with isoamylase were malto-oligosaccharides observed, and a sample that was treated solely with $\beta$-amylase showed the presence of only maltose as expected (data not shown). Additional samples were analyzed as summarized in Table 1.

indicative of the liberation of linear malto-oligosaccharides that were too long to remain in solution (>DP20). In the presence of iodine, such a sample exhibited significant absorbance with a $\lambda_{\max }$ of $\sim 537 \mathrm{~nm}$, revealing the liberation of linear maltooligosaccharides of DP up to $\sim 30$. $^{37}$

Matrix assisted laser desorption ionization mass spectrometry showed that that debranched $S$. venezuelae $\alpha$-glucan and rabbit liver glycogen had mean DPs (mass average) of 7.3 and 10.8, respectively (Figure 4 and Table 1 ), the latter consistent with published values. ${ }^{29}$ The narrower distribution of DPs in $\alpha$ glucan compared with glycogen was also reflected in the standard deviations of their means of 1.8 and 4.6, respectively. The reliability of this analysis was independently verified using capillary electrophoresis (Figure 4). For example, the $S$. venezuelae material gave a value of $7.7 \pm 2.2$, which was similar to the value of $7.3 \pm 1.8$ obtained with mass spectrometry. Analysis of the materials from M. tuberculosis capsule and cytosol showed that they comprised DPs of $\sim 7$ that were similar not only to that of $S$. venezuelae, but also each other (Table 1). A similar value was obtained with material isolated from whole $M$. smegmatis colonies $(7.1 \pm 2.0)$. By contrast, the E. coli material had a longer mean DP of $11.0 \pm 2.9$ (Table 1), as expected for a classical glycogen. ${ }^{29}$

Polymer samples treated with pullulanase from either Bacillus acidopullulyticus or Klebsiella pneumonia also led to the cleavage of $\alpha-1,6$ linkages. However, the hydrolysis of $\alpha-1,4$ linkages also occurred such that glucose and maltose were the sole products after $24 \mathrm{~h}$. This side reaction and/or contamination of pullulanase preparations might explain the very narrow distribution of very short chain lengths reported in one study. ${ }^{16}$

The degree of branching (the ratio between the number of $\alpha$ 1,6 linkages and the total number of linkages) can be calculated from the reciprocal of the mean chain DP. This gave 0.14 and 0.09 for actinomycete $\alpha$-glucans and glycogens, respectively (Table 1). These values were independently verified by integrating the anomeric resonances observed using ${ }^{1} \mathrm{H}$ NMR spectroscopy (Table 1). Furthermore, the values were consistent with previous reports for materials isolated from mycobacteria based on either methylation analysis, which gave a degree of branching of $0.14,{ }^{10,17,36}$ or enzymatic analyses. ${ }^{34}$ 
The C Chain of S. venezuelae $\alpha$-Glucan has a DP of $\sim 9$. Classical glycogen is an arboreal (tree-like) polymer. From the sole $\mathrm{C}$ chain with its reducing end stem one or more $\alpha-1,6$ linked B chains that, in turn, bear further $\alpha-1,6$ linked branches. The extremities of the nonreducing ends of the polymer comprise A chains that do not themselves bear branches. The $\mathrm{C}$ chain is uniquely addressable through reduction with sodium borohydride leading to an increase in mass by $2 \mathrm{Da}$. The debranching of reduced $S$. venezuelae $\alpha$-glucan allowed the detection of small amounts of reduced linear maltooligosaccharides using mass spectrometry. For example, chains with a DP of 8 in nonreduced control samples gave the natural isotopic distribution of isotopologues (Figure 5). However, a

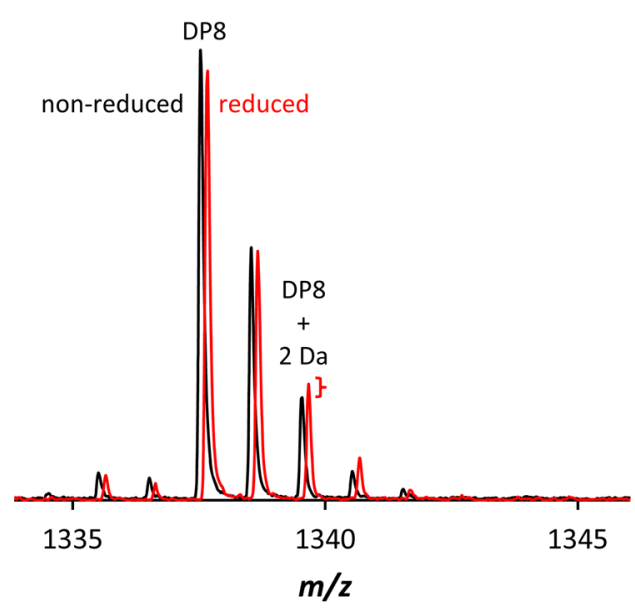

Figure 5. Detection of short C chains in $S$. venezuelae $\alpha$-glucan. The mass spectra show the isotopic distributions of the DP8 product of debranched $\alpha$-glucan with or without prior reduction. Without prior reduction (black), the natural abundance isotopic distribution of isotopologues was observable. With prior reduction with $\mathrm{NaBH}_{4}$ (red; displaced slightly along the horizontal axis for clarity), a small but significant relative enhancement of the isotopologue with two addition mass units $(1339.4 \mathrm{~m} / \mathrm{z}$ for DP8) was observed (highlighted by the bracket), indicative of the presence of small quantities of $\mathrm{C}$ chains of DP8 that had been reduced at their reducing ends. Spectra of products of DP4 to 14 were similarly recorded, and the absolute differences in the signals with two additional mass units for each DP allowed the chain length distribution of $\mathrm{C}$ chains to be calculated.

reduced sample showed a relative increase in the abundance of the isotopologue with an additional two mass units. Such reduced species must have been liberated $\mathrm{C}$ chains because malto-oligosaccharides are detected by mass spectrometry in samples of $\alpha$-glucan only when they are debranched. The reduced products had a mean $\mathrm{DP} \pm \mathrm{SD}$ of $9.1 \pm 2.5$ (Table 1 ). Therefore, the $\mathrm{C}$ chains were slightly longer than the average of $\sim 7$ of all chains within the polymer.

Internal Chain Length of Actinomycete $\alpha$-Glucan B Chains Is 4.6-5.0. To define the internal chain length of $B$ chains (i.e., between the branch point of $B$ chains and the furthest branch they bear as illustrated in Figure 3), $\alpha$-glucan samples were treated with $\beta$-amylase. This exoacting enzyme hydrolyzes maltose units from the nonreducing ends of $\mathrm{B}$ chains to within one or two glucosyl residues of the first branch point it encounters, depending on whether the external chain has an odd or even number of sugar residues. ${ }^{29,38}$ Given that there is no detectable odd/even bias within the polymer chains, the average overhang beyond the outermost branch after digestion is 1.5 glucosyl residues in length. By contrast, A chains are almost completely digested to give maltose and maltotriose (Figure 3). After treatment, the mean DPs of actinomycete $\alpha$-glucans of DP $\geq 4$ were 6.1-6.5 (Table 1 and Figure 4), showing that a typical B chain of seven residues bears a branch 4.6-5.0 residues from its own branch point (i.e., roughly half way along their length on their fourth or fifth residue). This contrasts with classical glycogens from rabbit liver and $E$. coli that have internal chain lengths of $\sim 7-8$ (Table 1).

$\alpha$-Glucan from S. venezuelae Has a Smaller A:BC Chain Ratio than Classical Glycogen. The ratio of the number of $A$ vs $B$ plus $C$ chains $(A: B C)$ of classical glycogen is typically close to $1 .{ }^{29}$ The A:BC chain ratio can be determined from the mean $\mathrm{DP}$ before and after treatment with $\beta$-amylase together with the extent of hydrolysis by this enzyme (where eq 2 is derived from eq 1 , which is also illustrated in Figure 3). The mean number of branches per $\mathrm{B} / \mathrm{C}$ chain in a polymer of finite size is essentially the $\mathrm{A}: \mathrm{BC}$ chain ratio plus one. For example, consider a $17 \mathrm{kDa}$ polymer consisting of 105 glucosyl residues in 15 chains with a mean DP of 7 (Figure S4). If each B and C chain bore only one branch (Figure S4A), the polymer would comprise 1 A chain, $13 \mathrm{~B}$ chains one $\mathrm{C}$ chain, giving an $\mathrm{A}: \mathrm{BC}$ chain ratio of close to zero (0.07). If the polymer comprised $B$ and $C$ chains that bore two chains each (Figure S4B), it would have eight A chains, six $\mathrm{B}$ chains, and $1 \mathrm{C}$ chain, giving an $\mathrm{A}: \mathrm{BC}$ chain ratio of close to 1 (1.14).

$\alpha$-Glucans were conspicuously less prone to hydrolysis by $\beta$ amylase $(21-33 \%$ glucose liberated) than E. coli classical glycogen ( 40-47\%) (Table 1). This was also evident from electron microscopy and dynamic light scattering (Table 1) that showed that only glycogen particles were significantly diminished in size. The A:BC chain ratio with $S$. venezuelae $\alpha$ glucan was $0.2 \pm 0.2$, giving $\sim 1.2 \pm 0.2$ branches per $\mathrm{B} / \mathrm{C}$ chain (Table 1). The values for both types of M. tuberculosis $\alpha$-glucan were a little higher at 0.6 and $\sim 1.6$, respectively. These values contrast with those of $0.8-0.9$ and 1.8-1.9, respectively, for classical glycogens (Table 1). ${ }^{29}$

GlgB Enzymes from Actinomycetes Generate Relatively Short Branches of DP 7-8. It might be expected that the branch length specificity of GlgB branching enzyme from actinomycetes reflects the shorter lengths present in the $\alpha$-glucans from these bacteria. The treatment of $\alpha$-1,4-linked amylose with a short or medium DP with M. tuberculosis GlgB branching enzyme led to a loss of absorbance in the presence of iodine (Figure S5), as previously described. ${ }^{39}$ The chain length specificity of this enzyme was then determined by exposing $\alpha$ maltose 1-phosphate to GlgE and GlgB to generate a branched $\alpha$-glucan product that could be debranched and subjected to mass spectrometry (Figure 6). The chains generated by $M$. tuberculosis $\mathrm{GlgB}$ in the presence of $M$. tuberculosis $\mathrm{GlgE}$ had a mean DP of $8.6 \pm 2.5$ (Table 2). This value was verified using capillary electrophoresis, which gave $8.0 \pm 3.1$ (Table 2). No branched products were observed when malto-octaose was exposed to GlgB suggesting this malto-oligosaccharide was not long enough to be branched. Mean values of between 7.2 and 8.5 were obtained when using S. coelicolor GlgE with GlgB from either M. tuberculosis or a number of other Mycobacterium and Streptomyces species (Table 2). This contrasts with chain DPs of $>10$ observed with branching enzymes from organisms that produce classical glycogen, such as that from E. coli. ${ }^{18,40}$ The actinomycete $\mathrm{GlgB}$ branching enzymes therefore generate relatively short branches, as predicted. 

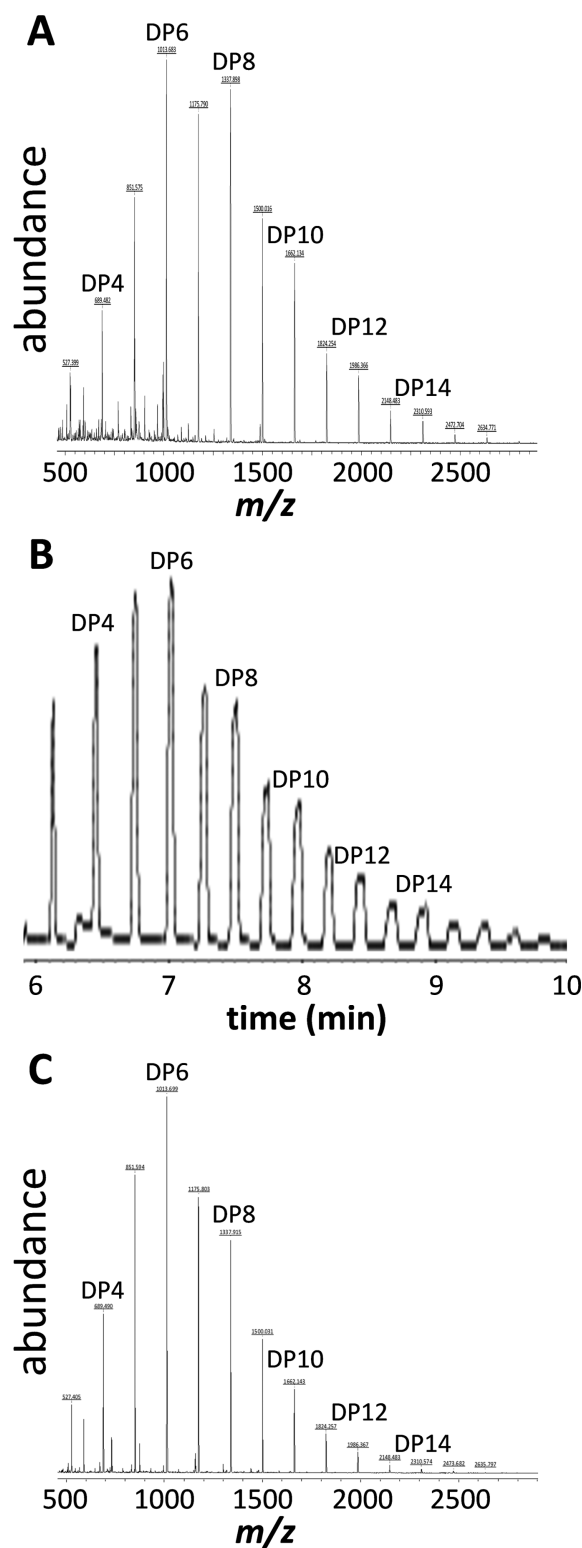

Figure 6. Branch length specificity of GlgB branching enzymes. The polymer, generated using $M$. tuberculosis GlgB, S. coelicolor GlgE, and $\alpha$-maltose 1-phosphate, was filtered to remove small maltooligosaccharides and then treated with isoamylase. The resulting debranched sample was then analyzed using either (A) MALDI mass spectrometry or (B) capillary electrophoresis. (C) A mass spectrum is also shown of a sample generated with GlgB enzyme from $S$. venezuelae. The mean branch lengths are shown in Table 1 .

M. tuberculosis GlgB Transfers a Nascent A Chain of DP $\sim 8$ to a Recipient Nascent C Chain of DP $\sim 9$. The mean chain DPs of the polymers generated in the presence of GlgB and GlgE would include all A, B, and C chains, where the $B$ chains would dominate, and the $C$ chains would be the least represented. We therefore devised an experiment to define the DP of nascent A chains that are transferred onto nascent $\mathrm{C}$ chains (Figure 7A). Malto-octaose (DP8) was first treated with GlgE and $\alpha$-maltose 1-phosphate to extend it by maltose units to give a series of products with an even number of glucosyl residues. The sample was then boiled, to denature the GlgE, followed by treatment with $\mathrm{GlgB}$ to introduce a single branch. The sample was boiled again, to denature the GlgB, reduced
Table 2. Branch Length Distributions Generated by GlgB Branching Enzymes in the Presence of GlgE

\begin{tabular}{|c|c|c|c|}
\hline \multirow[b]{2}{*}{ source of $\mathrm{GlgB}$} & \multirow[b]{2}{*}{ source of GlgE } & \multicolumn{2}{|c|}{ branch length $^{a}$} \\
\hline & & $\mathrm{MS}^{b}$ & $\mathrm{CE}^{c}$ \\
\hline M. tuberculosis & M. tuberculosis & $8.6 \pm 2.5$ & $8.0 \pm 3.1$ \\
\hline & S. coelicolor & $8.1 \pm 2.7$ & $7.5 \pm 3.5$ \\
\hline A chains & $\mathrm{na}^{d}$ & $8.3 \pm 1.9^{e}$ & $\mathrm{nd}^{f}$ \\
\hline odd length $\mathrm{C}$ chains & $\mathrm{na}^{d}$ & $9.0 \pm 2.9^{e}$ & $\mathrm{nd}^{f}$ \\
\hline M. tuberculosis CCDC5180 & S. coelicolor & $8.4 \pm 2.5$ & $\mathrm{nd}^{f}$ \\
\hline M. smegmatis $\mathrm{mc}^{2} 155$ & S. coelicolor & $8.5 \pm 2.5$ & $\mathrm{nd}^{f}$ \\
\hline S. venezuelae & S. coelicolor & $7.9 \pm 2.4$ & $\mathrm{nd}^{f}$ \\
\hline S. coelicolor isoform 1 & S. coelicolor & $7.2 \pm 2.2$ & $7.1 \pm 3.1$ \\
\hline
\end{tabular}

${ }^{a}$ Length distributions shown are mean \pm SD of products of DP $\geq 3$ of samples of malto-hexaose treated with GlgB, GlgE, and $\alpha$-maltose 1phosphate followed by isoamylase. ${ }^{b}$ Determined using mass spectrometry. ${ }^{c}$ Determined using capillary electrophoresis. ${ }^{d}$ Not applicable. ${ }^{e}$ The $\mathrm{A}$ and $\mathrm{C}$ chain length distributions were determined from a single branching event as shown in Figure $7 .{ }^{f}$ Not determined.

with $\mathrm{NaBH}_{4}$, and treated with isoamylase to cleave the $\alpha-1,6$ linkages. This would liberate reduced $\mathrm{C}$ chains and nonreduced A chains. All nonreduced species would be A chains. By contrast, the reduced C chains would be significantly contaminated with starting materials with an even number of residues that would also be reduced. GlgB does not discriminate between odd and even chain transfers. Therefore, reduced species that had an odd DP would be liberated C chains, with only a low level of contamination from hydrolytic side-products, as described below.

Mass spectrometry was used to quantify the abundance of reduced C chains that had an odd DP, giving a mean DP of 9.0 \pm 2.9 (Figure 7B and Table 2). For A chains, the distribution of nonreduced species with an even DP (mean DP $8.1 \pm 2.7$; data not shown) was similar to that of the odd species (mean DP 8.5 \pm 2.6 shown in Figure 7B) giving an overall mean DP of $8.3 \pm$ 2.7 (Table 2). This value was consistent with the DP of 8.1-8.6 generated in the presence of GlgE as described above (Table 2). The overall higher abundance of reduced over nonreduced chains was consistent with GlgB generating some hydrolytic products. From this, the hydrolysis to branching ratio was estimated to be $\sim 0.2: 1$ for such single branching events with short-chain substrates.

Branching by $M$. tuberculosis GlgB Is Strictly Intrachain. Branching enzymes act by transferring a segment from the nonreducing end of a malto-oligosaccharide to a hydroxyl group at the six position of a glucosyl residue along a maltooligosaccharide, via cleavage of an $\alpha-1,4$ linkage and a glycosylenzyme intermediate. ${ }^{41}$ Both intra- and interchain branching has been reported with GlgB enzymes. ${ }^{18,42,43}$ In order to establish whether M. tuberculosis GlgB carries out intra- and/or interchain transfers, we first generated ${ }^{13} \mathrm{C}$-labeled and unlabeled populations of malto-oligosaccharides (Figure 8 and Figure 9). This was possible by exposing unlabeled malto-tetraose (DP4) to GlgE in the presence of labeled or unlabeled $\alpha$-maltose 1-phosphate. These two populations of molecules were then boiled to denature the GlgE, mixed, boiled again, and then cooled, to allow any intermalto-oligosaccharide associations that might potentially occur to be formed at random. On exposure to GlgB, there would be three possible outcomes (Figure 7). If the branching reaction were intrachain, the masses of the products would be identical to those of the substrates. If it were interchain, the label would be redistributed 
A

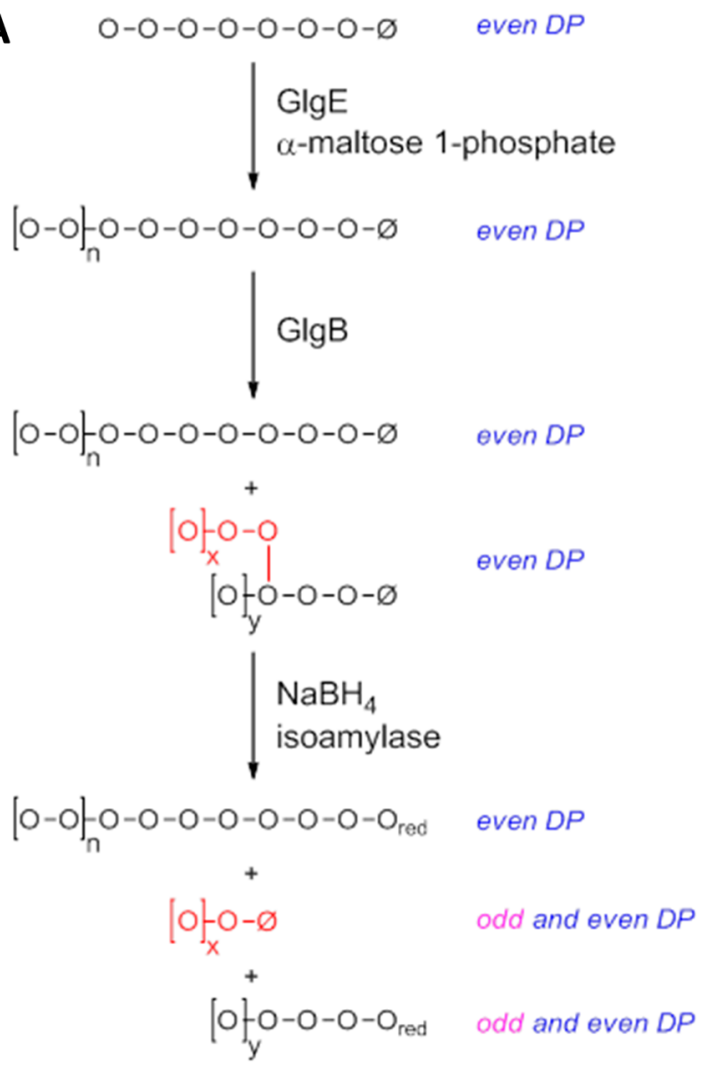

B

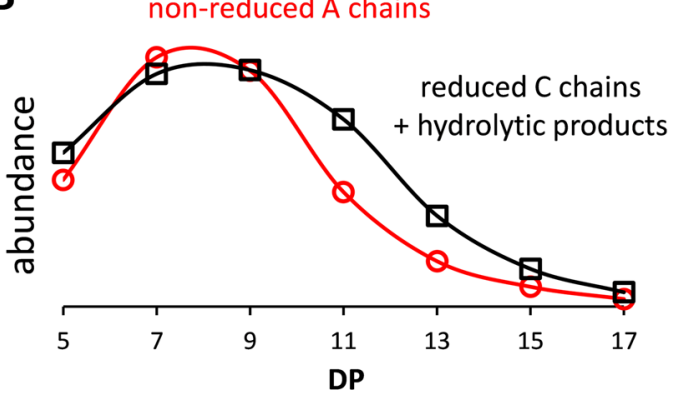

Figure 7. Branch length distributions of $\mathrm{A}$ and $\mathrm{C}$ chains generated by M. tuberculosis $\mathrm{GlgB}$ are similar. (A) The scheme shows the experimental design. Linear molecules and $C$ chains are shown in black, and $A$ chains are shown in red, where $\varnothing$ and $O_{\text {red }}$ refer to reducing end and reduced glucose residues, respectively. A population of malto-oligosaccharides with even DPs was generated with GlgE, malto-octaose, and $\alpha$-maltose 1-phosphate. After heat-denaturation of $\mathrm{GlgE}$, the sample was treated with GlgB. After heat-denaturation of $\mathrm{GlgB}$, the sample was reduced and treated with isoamylase to cleave the $\alpha-1,6$ linkages. (B) The final products from the experiment were subjected to mass spectrometry. The abundance of malto-oligosaccharide ions are plotted as a function of their degree of polymerization (DP). The quantification of reduced species took into account the natural abundance of isotopes from experimental mass spectra of debranched $S$. venezuelae $\alpha$-glucan. This was necessary because the larger the malto-oligosaccharide, the more naturally abundant species with $(m+2) / z$ were in control samples. Nonreduced species with an odd DP would have originated from A chains created by GlgB (plotted in red), while reduced species would have originated from $C$ chains and hydrolytic products (plotted in black).

in the products. If both types of reaction were to occur, there would be a partial redistribution of the label in the products.

It was possible to generate labeled and unlabeled starting materials with a DP of at least 20 (Figure 9). Closer inspection

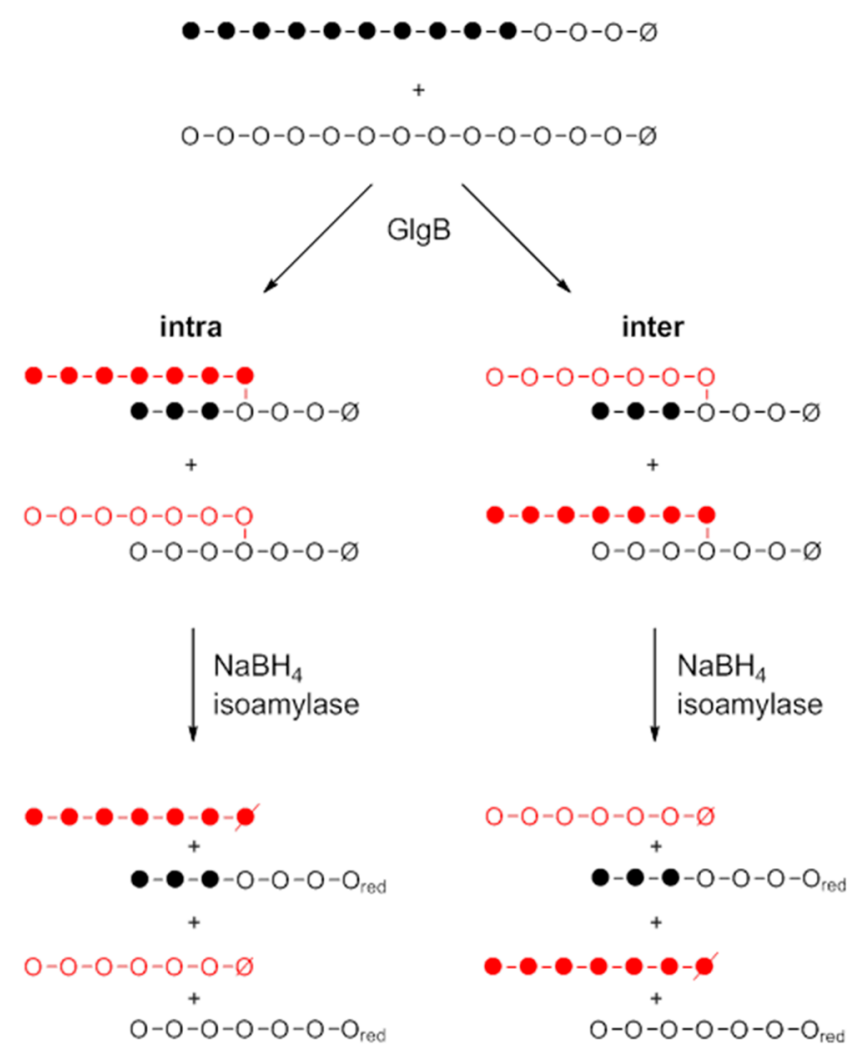

Figure 8. Experimental scheme to distinguish between intra- and interchain mechanisms of GlgB. Linear molecules and $\mathrm{C}$ chains are shown in black, and A chains are shown in red. Linear maltooligosaccharides were generated without or with labels by extending malto-tetraose with $M$. tuberculosis $\mathrm{GlgE}$ using either $\left[\mathrm{U}^{12} \mathrm{C}\right]$ or $\left[\mathrm{U}-{ }^{13} \mathrm{C}\right] \alpha$-maltose 1-phosphate, respectively (see Figure 9). A mixture of these materials was then exposed to $M$. tuberculosis GlgB to establish whether the distribution of the label was retained in the products or not (see Figure 10). The filled circles, $\varnothing$, and $\mathrm{O}_{\text {red }}$ refer to labeled, reducing end, and reduced glucosyl residues, respectively.

of the unlabeled starting material showed that it contained the expected natural abundance isotopic distribution (Figure 9B). By contrast, the labeled material was dominated by a species with the expected number of ${ }^{13} \mathrm{C}$ labels, together with some higher natural abundance isotopes associated largely with the four unlabeled glucosyl residues (Figure 9D). In addition, there was a low level of some species with one or two labels missing, reflecting the fact that the $\alpha$-maltose 1-phosphate was not quite $100 \%$ labeled.

Exposure of the mixed starting material to $M$. tuberculosis GlgB led to little obvious change in the overall distribution of chain lengths (Figure 10A). However, some hydrolysis had occurred because of a slight increase in the presence of species with an odd DP (Figure 10A) compared with the starting materials, which contained relatively little (Figure 9). For example, the unlabeled DP7 hydrolysis product had an abundance $8 \%$ that of the unlabeled DP6 species, showing that hydrolysis was a minor side reaction.

Most importantly, closer inspection of the main species with an even DP showed that the products contained the same distribution of label as the starting materials. For example, the DP16 species, which would be expected to include a product with a single branch point, had masses identical to those of the starting materials (Figure 10B) by being either fully unlabeled or with all but four glucosyl residues labeled. This is consistent 

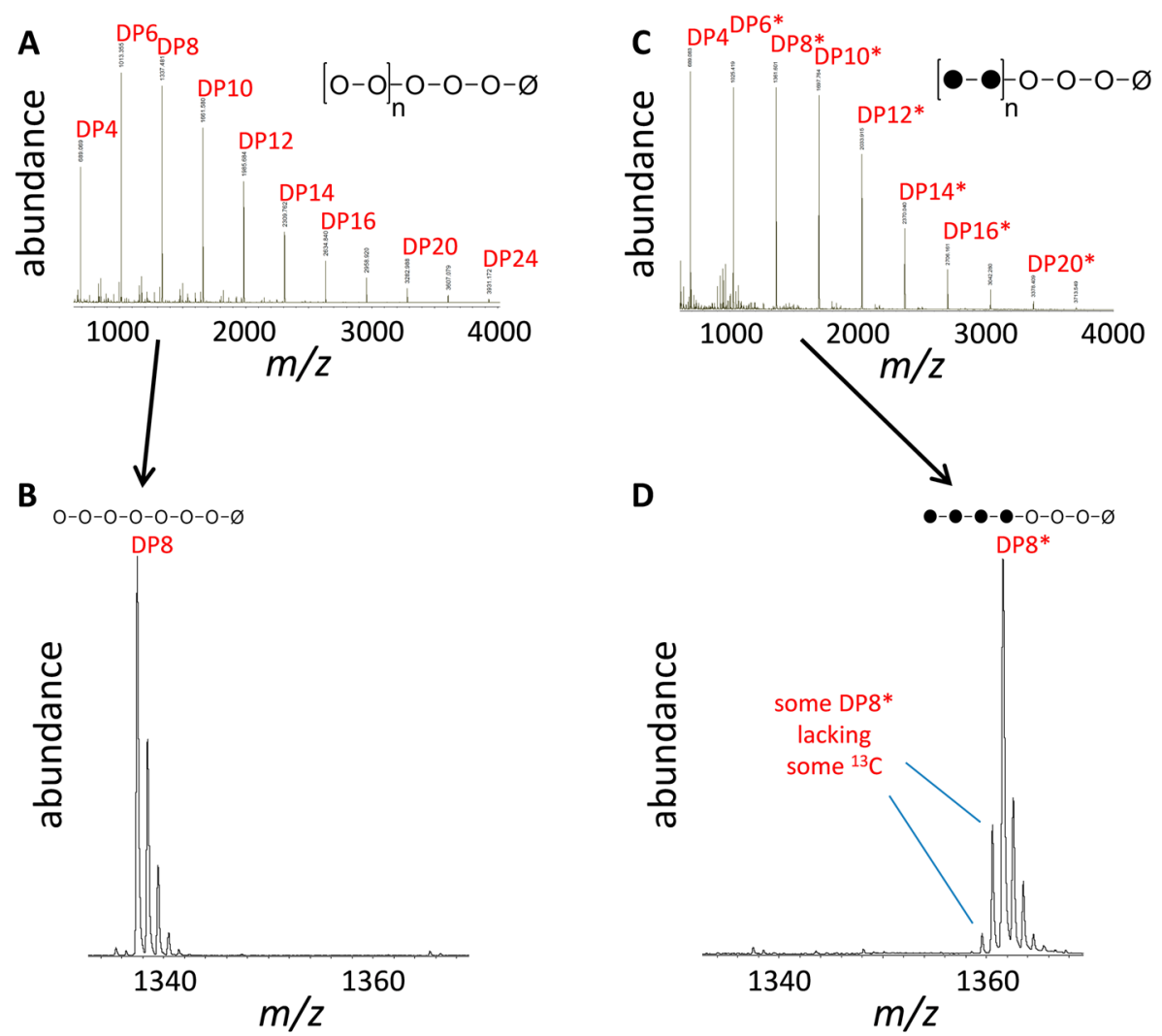

Figure 9. Enzymatic synthesis of labeled and unlabeled malto-oligosaccharides. Malto-tetraose (DP4) was extended by M. tuberculosis GlgE with either $\left[\mathrm{U}-{ }^{12} \mathrm{C}\right]$ or $\left[\mathrm{U}-{ }^{13} \mathrm{C}\right] \alpha$-maltose 1-phosphate as depicted in Figure 8 . The filled circles and $\varnothing$ in the structures refer to labeled and reducing end glucosyl residues, respectively. To stop the reaction, the samples were boiled to denature the enzyme. (A) A mass spectrum of unlabeled compounds showed the presence of a range of products. (B) The ions associated with each unlabeled product gave the expected natural abundance isotopic distribution, as illustrated by the DP8 product shown. (C) A mass spectrum of the labeled compounds also showed the presence of a range of products. (D) The ions associated with each labeled product showed the presence of the label, as illustrated by the DP8* product. The asterisk refers to the presence of ${ }^{13} \mathrm{C}$-labeled glucosyl residues observed in all but the original DP4 acceptor.

with strictly intrachain branching because interchain branching would have given additional species with intermediate amounts of label.

In order to confirm that branching had indeed occurred, the sample was reduced and then debranched (Figure 8). It was immediately apparent that the overall distribution of species was shifted to shorter DPs (Figure 10C) consistent with the cleavage of branch points. Closer inspection of the products confirmed that this was indeed the case. For example, the reduced, labeled DP8 species had the identical isotopic distribution (Figure 10D) as the starting material (Figure 9D), except for the addition of $2 \mathrm{Da}$ to all ions. This is consistent with starting material and liberated $\mathrm{C}$ chains (and likely some hydrolytic products). Furthermore, this shows that reduction was complete because no unreduced labeled species were detected. By contrast, the unlabeled DP8 product contained not only species with the same isotopic distribution as starting material plus $2 \mathrm{Da}$, but also nonreduced species amounting to over a quarter of the total unlabeled DP8 species (Figure 10D). The unreduced species can only have been derived from A chains formed by the branching reaction of GlgB. Such nonreduced species, with and without labels, were detected with a DP of between 5 and 10 giving a mean DP of $8.0 \pm 1.4$ for all liberated A chains, again consistent with the branching specificity experiments described above. These observations are therefore strongly supportive of a strictly intrachain mechanism.
Re-Evaluation of the Ability To Detect MaltoOligosaccharides Containing an $\alpha-1,6$ Branch Using Ion Mobility Mass Spectrometry. We previously interpreted the presence of two isomers of a malto-oligosaccharide with a DP of 11 in samples of amylose treated with M. tuberculosis GlgB as evidence for the ability to resolve linear and branched materials using ion-mobility mass spectrometry. ${ }^{25}$ Careful reevaluation of reaction mixtures with and without enzyme revealed that the relative abundance of the two observable isomers was in fact somewhat variable and, more critically, independent of GlgB (Figure S6). Reduction of a sample of linear malto-oligosaccharide (amylose 2800) with $\mathrm{NaBH}_{4}$ clearly showed the collapse of the two isomers of DP11 into one. This phenomenon resembles what we reported with malto-hexaose (DP6), which was interpreted as the presence of two conformers, where one is extended and the other forms the first complete turn of a left-handed helix. Each turn of the helix involves the formation of $\mathrm{O}(6)-\mathrm{O}(2)$ hydrogen bonds between glucosyl residues along the helical axis. Reduction of the reducing end would open up the terminal glucopyranose ring, disrupting the potential for such hydrogen bonding involving this residue. We therefore now interpret the presence of two isomers in the material with a DP of 11 in a similar way; it could adopt either a conformation with two complete turns of the helix or another more extended conformation. The variability in the relative abundance of the two isomers likely reflects very small differences in the stabilities of the two 


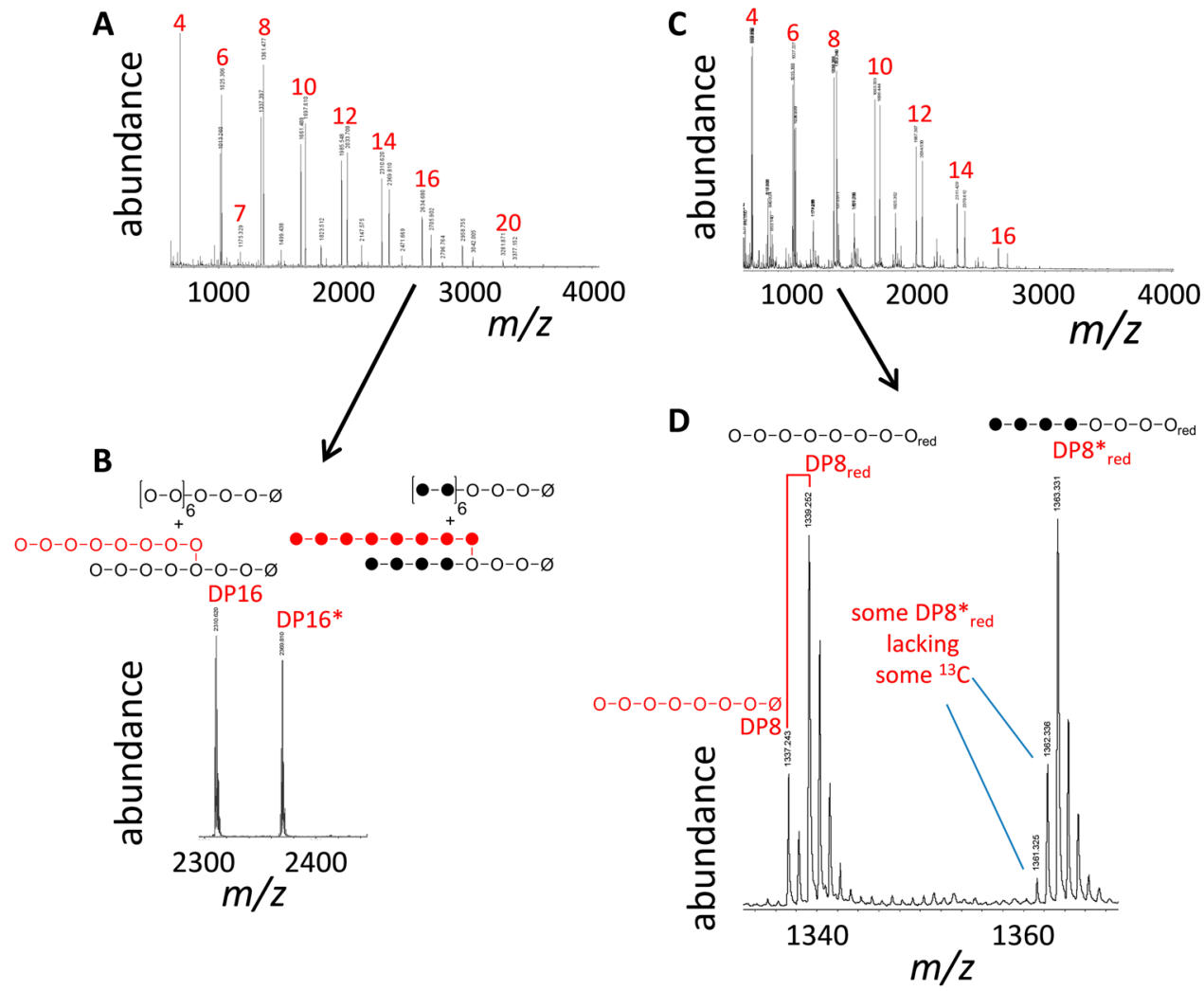

Figure 10. M. tuberculosis GlgB catalyzes intrachain branching. Labeled and nonlabeled compounds (see Figure 9) were mixed in equal proportions, boiled, cooled and treated with GlgB (see Figure 8 for experimental scheme). (A) A mass spectrum showed only minor changes in the profile of DP compared with starting materials (see Figure 9). (B) None of the products with an even DP showed any redistribution of ${ }^{13} \mathrm{C}$ labels, as illustrated with the DP16 product. The filled circles, $\varnothing$, and $\mathrm{O}_{\text {red }}$ in the structures refer to labeled, reducing end, and reduced glucosyl residues, respectively. Structures in black were derived from linear molecules and $\mathrm{C}$ chains, while those in the color red were derived from A chains. (C) The sample was reduced with $\mathrm{NaBH}_{4}$ and treated with isoamylase to hydrolyze all of the $\alpha-1,6$ linkages in order to confirm the presence of branches. The mass spectrum showed the loss of compounds with the highest DP, consistent with the cleavage of branches. (D) The reduced products illustrate how the reduced products $\left(\mathrm{DP}_{\text {red }}\right.$ and $\mathrm{DP} 8{ }_{\text {red }}$ ), derived from $\mathrm{C}$ chains and linear molecules, had the same isotopic distribution as the starting material, while the presence of nonlabeled, nonreduced product (DP8) showed that sample must have contained A chains generated by GlgB (see Figure 8 for the experimental scheme and the main text for further details).

conformations in the gas phase, and reduction would disrupt the formation of two complete helical turns.

GlgE Preferentially Extends A Chains over B and C Chains. GlgE must extend chains to attain the requisite length for GlgB to act. The B and C chains of an $\alpha$-glucan cannot be frequently extended by GlgE because the majority of B and $\mathrm{C}$ chains bear only one branch, and their lengths clearly remain unchanged after being formed by GlgB. To test this hypothesis, the activity of $M$. tuberculosis GlgE was determined with rabbit liver glycogen and $S$. venezuelae $\alpha$-glucan, which have A:BC chain ratios of 0.9 and 0.2 , respectively (Table 1 ). This was done by monitoring inorganic phosphate release from the donor substrate of GlgE, $\alpha$-maltose 1-phosphate. The $k_{\text {cat }} / K_{\mathrm{m}}$ of the enzyme was 6.4-fold higher with rabbit liver glycogen (Figure S7), consistent with GlgE exhibiting a preference for A chains. We then treated an equal mass of either oligosaccharides that contained a single branch, glycogen or $\alpha$-glucan with GlgE and ${ }^{13} \mathrm{C}$-labeled $\alpha$-maltose 1-phosphate (for example, see Figure S8). Mass spectrometry of debranched samples showed the chains were extended by 94,38 , and $18 \%$, respectively, again showing a correlation between the number of available $\mathrm{A}$ chains and the degree of extension.

Particles of $\alpha$-Glucan Generated by GlgE and GlgB Resemble Those Isolated from Cells. Polymers generated by $M$. tuberculosis $\mathrm{GlgE}$ and $\mathrm{GlgB}$ from $\alpha$-maltose 1-phosphate and malto-hexaose (DP6) resembled those isolated from cells according to electron microscopy (Figure 2). The mean diameter \pm SE of the synthetic material was about half $(32 \pm$ $2 \mathrm{~nm}$ ) that of the biological polymer isolated from $M$. tuberculosis, but their morphologies were similar. Similar sized particles were generated when the reaction was carried out with GlgE from $S$. coelicolor $(30 \pm 2 \mathrm{~nm}, n=40)$. Comparable diameters were also obtained when both $S$. coelicolor enzymes were used either with malto-hexaose $(29 \pm 5 \mathrm{~nm}, n=10)$ or without $(25 \pm 4 \mathrm{~nm}, n=10)$, showing that polymer initiation is possible without a primer.

\section{DISCUSSION}

GlgB enzymes from actinomycetes generate polymers in the presence of GlgE with DPs of typically $8.1 \pm 2.5$, which is reasonably similar to the typical DP of $7.2 \pm 1.9$ observed in polymer material isolated from cells. These are, to the best of our knowledge, among the shortest branch lengths reported. The shortest reported thus far for GH13 branching enzymes appear to be $7.9 \pm 3.4$ from Vibrio vulnificus ${ }^{44}$ and $8.0 \pm 2.6$ from Deinococcus geothermalis. ${ }^{45} \mathrm{We}$ and others ${ }^{18}$ have attempted to correlate the specificity of branching enzymes with their amino acid sequence but have failed to identify any obvious motif or domain responsible for any particular property. Nevertheless, the two N-terminal domains of 
actinomycete GlgB enzymes, which include a family 48 carbohydrate binding module, do appear to be important because chimeric enzymes have altered chain length specificities. $^{44,45}$

Interestingly, branching by M. tuberculosis GlgB was shown to be strictly intrachain. This implies that the nascent recipient chain, and by definition the remainder of the polymer, must bind very tightly and noncovalently to the enzyme until the nascent A chain is fully transferred to the recipient chain via a glycosyl-enzyme intermediate. We and others ${ }^{41}$ have observed that branching enzymes can generate some hydrolytic byproducts. This was more prevalent with short substrates, implying fewer potential contacts with the protein lead to weaker binding. With the M. tuberculosis enzyme at least, it would appear that water, rather than another polymer chain, acts as the acceptor after premature dissociation of the original recipient chain.

We have shown that the internal chain length of $\alpha$-glucan is shorter than for classical glycogen. Many previous studies on glycogens have estimated internal chain lengths based solely on the degree of hydrolysis by $\beta$-amylase. This is inappropriate given that glycogen contains almost as many $\mathrm{A}$ chains and $\mathrm{B}$ chains, and A chains are almost completely digested. ${ }^{38}$ This artificially decreases the calculated internal chain lengths to such an extent that they would often not be long enough to support the degree of branching that is known to occur. In order to avoid this problem, we directly determined the length of $\mathrm{B}$ chains with mass spectrometry after treatment with $\beta$ amylase.

It is now possible to conceive of how $\alpha$-glucans are generated in organisms such as $S$. venezuelae (Figure 11), which possesses the GlgE pathway and no other $\alpha$-glucan pathway. ${ }^{8}$ It is known that GlgE can initiate polymer synthesis without any primer, such as glycogenin or a malto-oligosaccharide. It does this by slowly hydrolyzing $\alpha$-maltose 1-phosphate to give maltose, which in turn is slowly extended to give malto-tetraose, one of its preferred acceptor substrates. ${ }^{3,19}$ As soon as the maltooligosaccharide attains a length of $\sim 16$ glucosyl residues, GlgB will generate the first branch giving an $\mathrm{A}$ and a $\mathrm{C}$ chain of DP 8-9. GlgE will then preferentially extend the A chain until it is long enough to undergo branching to generate a new A chain and the first $B$ chain. The minimum length that seems to be transferred can occasionally be three but is more typically four or more in vivo and in vitro (Figure 4 and Figure 6). Each new A chain forms a branch as close as three residues from the branch point of the recipient chain, but more often on the fourth or fifth. By contrast, the E. coli enzyme has been reported to prefer the third residue for the first branch to be formed. ${ }^{18}$ GlgE and GlgB will continue to extend and create new A chains, respectively. Normally, a newly formed B chain will not be long enough to allow a second branch to be introduced by GlgB. However, GlgE appears to occasionally extend a B chain and, when it attains sufficient length, GlgB introduces a second branch into this chain. These additional events ultimately generate the arboreal structure of the $\alpha$-glucan. However, the introduction of a second branch in a B chain is not a frequent event because the mean number of branches per $B$ chain is $1.2-1.6$, considerably less than the value of $1.8-1.9$ routinely observed in classical glycogens. Indeed the A:BC chain ratios for $\alpha$-glucan are the smallest that have, to our knowledge, been reported. $^{29}$

Importantly, the majority of chains in an $\alpha$-glucan are $\mathrm{B}$ chains. Therefore, the overall mean linear chain DP of an $\alpha$ -

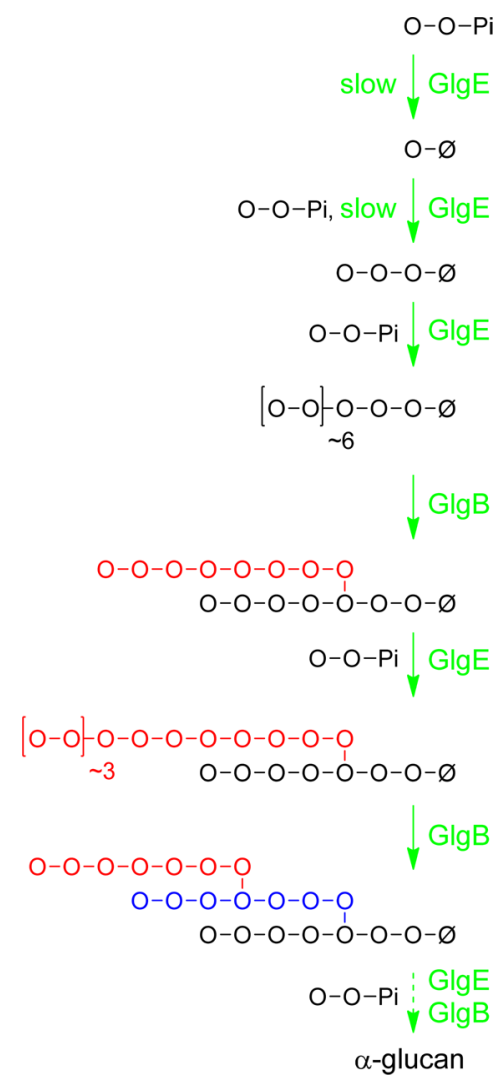

Figure 11. Scheme of $\alpha$-glucan assembly by GlgE and GlgB. Linear molecules and $\mathrm{C}$ chains are shown in black, $\mathrm{A}$ chains in red, and $\mathrm{B}$ chains in blue. The $\varnothing$ and $\mathrm{Pi}$ in the structures refer to the reducing end glucose residue and phosphate, respectively.

glucan must be principally defined by GlgB. This is because the length of each $B$ chain is initially defined by the recipient chain length specificity of $\mathrm{GlgB}$. We have established that $M$. tuberculosis GlgB creates $\mathrm{C}$ chains of $\mathrm{DP} \sim 9$ Table 1 and Figure 5), which matches that observed in the polymer isolated from cells. It is reasonable to assume that it also generates B chains of a length that matches those observed in the polymer of $\sim 7-8$. If GlgE readily extended B chains, one would expect that the mean linear chain DP in the polymer would be much greater, but this is clearly not the case. Rather, it would appear that the occasional extension of individual B chains by GlgE simply leads to $\mathrm{GlgB}$ acting again, significantly restricting any potential to extend $\mathrm{B}$ chains within the polymer. Consistent with this, the branch lengths generated by GlgB in vitro are not obviously influenced by the presence or absence of GlgE in our in vitro assays.

GlgE is capable of not only extending malto-oligosaccharides with $\alpha$-maltose 1-phosphate but also moving maltosyl units between them in a disproportionation reaction. ${ }^{3,19}$ Perhaps this second activity is important to prevent any chains from being excessively extended because linear malto-oligosaccharides with a DP of $>20$ have limited solubility in aqueous conditions.

Having previously used a genetic approach to show that the GlgE pathway is both necessary and sufficient for the biosynthesis of $\alpha$-glucan in $S$. venezuelae, we have now shown that $G \operatorname{lgE}$ and $\mathrm{GlgB}$ are sufficient to generate the polymer from solely $\alpha$-maltose 1-phosphate in vitro. Importantly, the $\alpha$-glucans isolated from the cytosol and capsule of $M$. tuberculosis were very similar not only to each other, but also to that from $S$. venezuelae, which is derived exclusively from the 
GlgE pathway. Although others have reported differences between the two mycobacterial materials, ${ }^{15,16}$ such as in optical rotation, particle size, and hydrolytic susceptibility, these differences are subtle and could reflect differences in the way in which the materials are isolated. The similarity in the structures of the cytosolic and capsular materials implies that they could be synthesized solely by the GlgE pathway. It therefore remains to be established what role GlgA and Rv3032 have in the biosynthesis of $\alpha$-glucan polymers in mycobacteria, despite genetic evidence suggesting they could have a direct role. $^{15}$ Furthermore, how a kDa-MDa dendrimer with a diameter of tens of nanometer is exported from a mycobacterial cell remains unclear, since secretion via vesicles appears to have been ruled out. ${ }^{46}$

\section{ASSOCIATED CONTENT}

\section{S Supporting Information}

The Supporting Information is available free of charge on the ACS Publications website at DOI: 10.1021/acs.biochem.6b00209.

NMR spectra of $\alpha$-glucan, calibration curve for pulse field gradient NMR spectroscopy, UV/visible absorbance spectra of iodine complexes, cartoons of theoretical structures, ion mobility mass spectra, and kinetics and mass spectrometric analysis of polymer extension by GlgE (PDF)

\section{AUTHOR INFORMATION}

\section{Corresponding Author}

*Telephone: +44 1603 450741. E-mail: stephen.bornemann@ jic.ac.uk.

\section{Funding}

This work was supported by the United Kingdom Biotechnology and Biological Sciences Research Council (Responsive Mode [BB/I012850/1], Doctoral Training Partnership [BB/ J014524/1], and Institute Strategic Programme [BB/J004561/ 1] grants) and the John Innes Foundation.

\section{Notes}

The authors declare no competing financial interest.

\section{ACKNOWLEDGMENTS}

We thank Govind Chandra for assistance with bioinformatics, Gerhard Saalbach for assistance with mass spectrometry, Jayne Watson for carrying out preliminary experiments, John (Jack) Thompson for the gift of the $\left[\mathrm{U}^{13} \mathrm{C}\right] \alpha$-maltose 1-phosphate, and Alison Smith, Keith Chater, and Rob Field for helpful discussions.

\section{ABBREVIATIONS}

$\mathrm{DP}$, degree of polymerization of linear $\alpha$-1,4-linked chains

\section{REFERENCES}

(1) Preiss, J. (2009) Glycogen biosynthesis, in The Encyclopedia of Microbiology Vol. 5 (Schaechter, M., Ed.) pp 145-158, Elsevier, Oxford, U.K.

(2) Chandra, G., Chater, K. F., and Bornemann, S. (2011) Unexpected and widespread connections between bacterial glycogen and trehalose metabolism. Microbiology 157, 1565-1572.

(3) Kalscheuer, R., Syson, K., Veeraraghavan, U., Weinrick, B., Biermann, K. E., Liu, Z., Sacchettini, J. C., Besra, G., Bornemann, S., and Jacobs, W. R. (2010) Self-poisoning of Mycobacterium tuberculosis by targeting GlgE in an $\alpha$-glucan pathway. Nat. Chem. Biol. 6, 376384.

(4) Elbein, A. D., Pastuszak, I., Tackett, A. J., Wilson, T., and Pan, Y. T. (2010) Last step in the conversion of trehalose to glycogen: a mycobacterial enzyme that transfers maltose from maltose 1phosphate to glycogen. J. Biol. Chem. 285, 9803-9812.

(5) Bornemann, S. (2016) $\alpha$-Glucan biosynthesis and the GlgE pathway in Mycobacterium tuberculosis. Biochem. Soc. Trans. 44, 68-73.

(6) Stam, M. R., Danchin, E. G. J., Rancurel, C., Coutinho, P. M., and Henrissat, B. (2006) Dividing the large glycoside hydrolase family 13 into subfamilies: towards improved functional annotations of $\alpha$ amylase-related proteins. Protein Eng., Des. Sel. 19, 555-562.

(7) Lombard, V., Golaconda Ramulu, H., Drula, E., Coutinho, P. M., and Henrissat, B. (2014) The carbohydrate-active enzymes database (CAZy) in 2013. Nucleic Acids Res. 42, D490-495.

(8) Miah, F., Bibb, M. J., Barclay, J. E., Findlay, K. C., and Bornemann, S. (2016) Developmental delay in a Streptomyces venezuelae glgE null mutant is associated with the accumulation of $\alpha$ maltose 1-phosphate, Microbiology 162, DOI: 10.1099/mic.0.000296.

(9) Jackson, M., and Brennan, P. J. (2009) Polymethylated polysaccharides from Mycobacterium species revisited. J. Biol. Chem. 284, 1949-1953.

(10) Lemassu, A., and Daffé, M. (1994) Structural features of the exocellular polysaccharides of Mycobacterium tuberculosis. Biochem. J. 297, 351-357.

(11) Schwebach, J. R., Glatman-Freedman, A., Gunther-Cummins, L., Dai, Z. D., Robbins, J. B., Schneerson, R, and Casadevall, A. (2002) Glucan is a component of the Mycobacterium tuberculosis surface that is expressed in vitro and in vivo. Infect. Immun. 70, 2566-2575.

(12) Cywes, C., Hoppe, H. C., Daffé, M., and Ehlers, M. R. W. (1997) Nonopsonic binding of Mycobacterium tuberculosis to complement receptor type 3 is mediated by capsular polysaccharides and is strain dependent. Infect. Immun. 65, 4258-4266.

(13) Gagliardi, M. C., Lemassu, A., Teloni, R., Mariotti, S., Sargentini, V., Pardini, M., Daffé, M., and Nisini, R. (2007) Cell wall-associated $\alpha$ glucan is instrumental for Mycobacterium tuberculosis to block CD1 molecule expression and disable the function of dendritic cell derived from infected monocyte. Cell. Microbiol. 9, 2081-2092.

(14) Geurtsen, J., Chedammi, S., Mesters, J., Cot, M., Driessen, N. N., Sambou, T., Kakutani, R., Ummels, R., Maaskant, J., Takata, H., Baba, O., Terashima, T., Bovin, N., Vandenbroucke-Grauls, C. M. J. E., Nigou, J., Puzo, G., Lemassu, A., Daffé, M., and Appelmelk, B. J. (2009) Identification of mycobacterial $\alpha$-glucan as a novel ligand for DC-SIGN: involvement of mycobacterial capsular polysaccharides in host imune modulation. J. Immunol. 183, 5221-5231.

(15) Sambou, T., Dinadayala, P., Stadthagen, G., Barilone, N., Bordat, Y., Constant, P., Levillain, F., Neyrolles, O., Gicquel, B., Lemassu, A., Daffé, M., and Jackson, M. (2008) Capsular glucan and intracellular glycogen of Mycobacterium tuberculosis: biosynthesis and impact on the persistence in mice. Mol. Microbiol. 70, 762-774.

(16) Dinadayala, P., Sambou, T., Daffé, M., and Lemassu, A. (2008) Comparative structural analyses of the $\alpha$-glucan and glycogen from Mycobacterium bovis. Glycobiology 18, 502-508.

(17) Ortalo-Magné, A., Dupont, M. A., Lemassu, A., Andersen, A. B., Gounon, P., and Mamadou, D. (1995) Molecular composition of the outermost capsular material of the tubercle bacillus. Microbiology 141, $1609-1620$.

(18) Sawada, T., Nakamura, Y., Ohdan, T., Saitoh, A., Francisco, P. B., Jr., Suzuki, E., Fujita, N., Shimonaga, T., Fujiwara, S., Tsuzuki, M., Colleoni, C., and Ball, S. (2014) Diversity of reaction characteristics of glucan branching enzymes and the fine structure of $\alpha$-glucan from various sources. Arch. Biochem. Biophys. 562, 9-21.

(19) Syson, K., Stevenson, C. E. M., Rejzek, M., Fairhurst, S. A., Nair, A., Bruton, C. J., Field, R. A., Chater, K. F., Lawson, D. M., and Bornemann, S. (2011) Structure of a Streptomyces maltosyltransferase GlgE: a homologue of a genetically validated anti-tuberculosis target. J. Biol. Chem. 286, 38298-38310.

(20) Yam, K. C., D’Angelo, I., Kalscheuer, R., Zhu, H., Wang, J.-X., Snieckus, V., Ly, L. H., Converse, P. J., Jacobs, W. R., Jr., Strynadka, N., 
and Eltis, L. D. (2009) Studies of a ring-cleaving dioxygenase illuminate the role of cholesterol metabolism in the pathogenesis of Mycobacterium tuberculosis. PLoS Pathog. 5, e1000344.

(21) Sani, M., Houben, E. N. G., Geurtsen, J., Pierson, J., de Punder, K., van Zon, M., Wever, B., Piersma, S. R., Jiménez, C. R., Daffé, M., Appelmelk, B. J., Bitter, W., van der Wel, N., and Peters, P. J. (2010) Direct visualization by cryo-EM of the mycobacterial capsular layer: a labile structure containing ESX-1-secreted proteins. PLoS Pathog. 6, e1000794.

(22) Miah, F., Koliwer-Brandl, H., Rejzek, M., Field, R. A., Kalscheuer, R., and Bornemann, S. (2013) Flux through trehalose synthase flows from trehalose to the alpha anomer of maltose in mycobacteria. Chem. Biol. 20, 487-492.

(23) Miller, M. C., Klyosov, A., Platt, D., and Mayo, K. H. (2009) Using pulse field gradient NMR diffusion measurements to define molecular size distributions in glycan preparations. Carbohydr. Res. 344, 1205-1212.

(24) Kieser, T., Bibb, M. J., Buttner, M. J., Chater, K. F., and Hopwood, D. A. (2000) Practical Streptomyces Genetics, The John Innes Foundation, Norwich, United Kingdom.

(25) Rashid, A. M., Saalbach, G., and Bornemann, S. (2014) Discrimination of large maltooligosaccharides from isobaric dextran and pullulan using ion mobility mass spectrometry. Rapid Commun. Mass Spectrom. 28, 191-199.

(26) McCleary, B. V., Gibson, T. S., and Mugford, D. C. (1997) Measurement of total starch in cereal products by amyloglucosidase- $\alpha$ amylase method: collaborative study. J. AOAC Int. 80, 571-579.

(27) Guzman, L. M., Belin, D., Carson, M. J., and Beckwith, J. (1995) Tight regulation, modulation and high-level expression by vectors containing the arabinose $\mathrm{P}_{\mathrm{BAD}}$ promotor. J. Bacteriol. 177, 4121-4130.

(28) Syson, K., Stevenson, C. E. M., Rashid, A. M., Saalbach, G., Tang, M., Tuukkanen, A., Svergun, D. I., Withers, S. G., Lawson, D. M., and Bornemann, S. (2014) Structural insight into how Streptomyces coelicolor maltosyl transferase GlgE binds $\alpha$-maltose 1-phosphate and forms a maltosyl-enzyme intermediate. Biochemistry 53, 2494-2504.

(29) Manners, D. J. (1991) Recent developments in our understanding of glycogen structure. Carbohydr. Polym. 16, 37-82.

(30) Dinadayala, P., Lemassu, A., Granovski, P., Cérantola, S., Winter, N., and Daffé, M. (2004) Revisiting the structure of the anti-neoplastic glucans of Mycobacterium bovis Bacille Calmette-Guérin - Structural analysis of the extracellular and boiling water extract-derived glucans of the vaccine substrains. J. Biol. Chem. 279, 12369-12378.

(31) Bittencourt, V. C. B., Figueiredo, R. T., da Silva, R. B., MouraoSa, D. S., Fernandez, P. L., Sassaki, G. L., Mulloy, B., Bozza, M. T., and Barreto-Bergter, E. (2006) An $\alpha$-glucan of Pseudallescheria boydii is involved in fungal phagocytosis and toll-like receptor activation. J. Biol. Chem. 281, 22614-22623.

(32) Tan, I., Flanagan, B. M., Halley, P. J., Whittaker, A. K., and Gidley, M. J. (2007) A method for estimating the nature and relative proportions of amorphous, single, and double-helical components in starch granules by $\mathrm{C}^{13} \mathrm{CP} / \mathrm{MAS}$ NMR. Biomacromolecules 8 , 885891.

(33) Ryu, J.-H., Drain, J., Kim, J. H., McGee, S., Gray-Weale, A., Waddington, L., Parker, G. J., Hargreaves, M., Yoo, S.-H., and Stapleton, D. (2009) Comparative structural analyses of purified glycogen particles from rat liver, human skeletal muscle and commercial preparations. Int. J. Biol. Macromol. 45, 478-482.

(34) Antoine, A. D., and Tepper, B. S. (1969) Characterization of glycogens from mycobacteria. Arch. Biochem. Biophys. 134, 207-213.

(35) van de Weerd, R., Berbís, M. A., Sparrius, M., Maaskant, J. J., Boot, M., Paauw, N. J., de Vries, N., Boon, L., Baba, O., Cañada, F. J., Geurtsen, J., Jiménez-Barbero, J., and Appelmelk, B. J. (2015) A murine monoclonal antibody to glycogen: characterization of epitopefine specificity by saturation transfer difference (STD) NMR spectroscopy and its use in mycobacterial capsular $\alpha$-glucan research. ChemBioChem 16, 977-989.

(36) Misaki, A., and Yukawa, S. (1966) Studies on cell walls of Mycobacteria. II. Constitution of polysaccharides from BCG cell walls. J. Biochem.(Tokyo) 59, 511-520.
(37) Bailey, J. M., and Whelan, W. J. (1961) Physical properties of starch. 1. Relationship between iodine stain and chain length. J. Biol. Chem. 236, 969-973.

(38) Summer, R, and French, D. (1956) Action of $\beta$-amylase on branched oligosaccharides. J. Biol. Chem. 222, 469-477.

(39) Garg, S. K., Alam, M. S., Kishan, K. V. R., and Agrawal, P. (2007) Expression and characterization of $\alpha$ - $(1,4)$-glucan branching enzyme Rv1326c of Mycobacterium tuberculosis H37Rv. Protein Expression Purif. 51, 198-208.

(40) Guan, H. P., Li, P., ImparlRadosevich, J., Preiss, J., and Keeling, P. (1997) Comparing the properties of Escherichia coli branching enzyme and maize branching enzyme. Arch. Biochem. Biophys. 342, 92-98.

(41) Pal, K., Kumar, S., Sharma, S., Garg, S. K., Alam, M. S., Xu, H. E., Agrawal, P., and Swaminathan, K. (2010) Crystal structure of full length Mycobacterium tuberculosis $\mathrm{H} 37 \mathrm{Rv}$ glycogen branching enzyme: insights of $\mathrm{N}$-terminal $\beta$-sandwich in substrate specificity and enzymatic activity. J. Biol. Chem. 285, 20897-20903.

(42) Hernández, J. M., Gaborieau, M., Castignolles, P., Gidley, M. J., Myers, A. M., and Gilbert, R. G. (2008) Mechanistic investigation of a starch-branching enzyme using hydrodynamic volume SEC analysis. Biomacromolecules 9, 954-965.

(43) Roussel, X., Lancelon-Pin, C., Vikso-Nielsen, A., Rolland-Sabaté, A., Grimaud, F., Potocki-Véronèse, G., Buléon, A., Putaux, J.-L., and D'Hulst, C. (2013) Characterization of substrate and product specificity of the purified recombinant glycogen branching enzyme of Rhodothermus obamensis. Biochim. Biophys. Acta, Gen. Subj. 1830, 2167-2177.

(44) Jo, H.-J., Park, S., Jeong, H.-G., Kim, J.-W., and Park, J.-T. (2015) Vibrio vulnificus glycogen branching enzyme preferentially transfers very short chains: N1 domain determines the chain length transferred. FEBS Lett. 589, 1089-1094.

(45) Palomo, M., Kralj, S., van der Maarel, M. J. E. C., and Dijkhuizen, L. (2009) The unique branching patterns of Deinococcus glycogen branching enzymes are determined by their N-terminal domains. Appl. Environ. Microbiol. 75, 1355-1362.

(46) Prados-Rosales, R., Baena, A., Martinez, L. R., Luque-Garcia, J., Kalscheuer, R., Veeraraghavan, U., Camara, C., Nosanchuk, J. D., Besra, G. S., Chen, B., Jimenez, J., Glatman-Freedman, A., Jacobs, W. R., Jr., Porcelli, S. A., and Casadevall, A. (2011) Mycobacteria release active membrane vesicles that modulate immune responses in a TLR2dependent manner in mice. J. Clin. Invest. 121, 1471-1483. 